\title{
Lorentz and CPT violation in partons
}

\author{
V. Alan Kostelecký, ${ }^{a}$ Enrico Lunghi, ${ }^{a}$ Nathan Sherrill ${ }^{a}$ and A.R. Vieira ${ }^{b}$ \\ ${ }^{a}$ Physics Department, Indiana University, \\ Bloomington, IN 47405, U.S.A. \\ ${ }^{b}$ Universidade Federal do Triângulo Mineiro, Campus Iturama, \\ 38280-000, Iturama $M G$, Brazil \\ E-mail: kostelec@indiana.edu, elunghi@indiana.edu, nlsherri@iu.edu, \\ alexandre.vieira@uftm.edu
}

ABSTRACT: A framework is presented for the factorization of high-energy hadronic processes in the presence of Lorentz and CPT violation. The comprehensive effective field theory describing Lorentz and CPT violation, the Standard-Model Extension, is used to demonstrate factorization of the hadronic tensor at leading order in electroweak interactions for deep inelastic scattering and for the Drell-Yan process. Effects controlled by both minimal and nonminimal coefficients for Lorentz violation are explored, and the equivalent parton-model description is derived. The methodology is illustrated by determining cross sections and studying estimated attainable sensitivities to Lorentz violation using real data collected at the Hadronen-Elektronen Ring Anlage and the Large Hadron Collider and simulated data for the future US-based electron-ion collider.

KEYWORDS: Deep Inelastic Scattering (Phenomenology)

ArXIV EPRINT: 1911.04002 


\section{Contents}

1 Introduction 1

2 Framework 3

3 Deep inelastic scattering 5

3.1 Factorization of the hadronic tensor 5

$\begin{array}{lll}3.2 & \text { The operator product expansion } & 10\end{array}$

$\begin{array}{lll}3.3 & \text { Minimal } c \text {-type coefficients } & 14\end{array}$

$\begin{array}{ll}3.4 & \text { Nonminimal } a^{(5)} \text {-type coefficients } \\ 3.5 & 18\end{array}$

$\begin{array}{ll}3.5 & \text { Estimated attainable sensitivities }\end{array}$

4 The Drell-Yan process $\quad 27$

4.1 Factorization of the hadronic tensor 28

4.2 Minimal $c$-type coefficients 30

4.3 Nonminimal $a^{(5)}$-type coefficients 33

4.4 Estimated attainable sensitivities and comparison with DIS 35

$\begin{array}{lll}5 & \text { Summary } & 37\end{array}$

\section{Introduction}

Deep inelastic scattering (DIS) and the Drell-Yan (DY) process are key tools in the study of quantum chromodynamics (QCD). The DIS cross section for electron-proton scattering depends only weakly on momentum transfer [1,2], and the scaling invariance of the associated form factors [3] implies that nucleons contain partons [4]. The DY process [5], which involves the production and decay of vector bosons in hadron collisions, is related by crossing symmetry to DIS and provides complementary information about the parton distribution functions (PDFs) [6]. Both DIS and the DY process play a crucial role in investigations of perturbative QCD and can serve as probes for physics beyond the Standard Model (SM) [7].

One interesting prospect for experimental signals beyond the SM is minuscule violations of Lorentz and CPT symmetry, which may originate from the Planck scale in an underlying theory combining quantum physics and gravity such as strings [8-10]. Over the last two decades, this idea has been extensively tested via precision tests with gravity and with many SM particles and interactions [11], but comprehensive studies directly involving quarks remain challenging due primarily to complications in interpreting hadronic results in terms of the underlying QCD degrees of freedom. In this work, we develop factorization techniques for hadronic processes in the presence of Lorentz and CPT violation and apply them to DIS and the DY process, using the results to estimate attainable sensitivities in certain experiments at the Hadronen-Elektronen Ring Anlage (HERA) [12], at the 
electron-ion collider (EIC) proposed for Thomas Jefferson National Laboratory (JLab) or Brookhaven National Laboratory (BNL) [12], and at the Large Hadron Collider (LHC) [13].

The methodology adopted in this work is grounded in effective field theory, which provides a quantitative description of tiny effects emerging from distances below direct experimental resolution [14]. The comprehensive realistic effective field theory for Lorentz violation, called the Standard-Model Extension (SME) [15-17], is obtained by adding all Lorentz-violating terms to the action for general relativity coupled to the SM. Since violation of CPT symmetry implies Lorentz violation in realistic effective field theory $[15,18]$, the SME also characterizes general effects of CPT violation. Any given Lorentz-violating term is constructed as the coordinate-independent contraction of a coefficient for Lorentz violation with a Lorentz-violating operator. The operators can be classified according to mass dimension $d$, and terms with $d \leq 4$ in Minkowski spacetime yield a theory called the minimal SME that is power-counting renormalizable. Reviews of the SME can be found in, for example, refs. [11, 19-21].

We concentrate here on evaluating the effects on DIS and the DY process of coefficients for Lorentz violation controlling spin-independent SME operators involving the $u$ and $d$ quarks and having mass dimension four and five. The former are minimal SME operators preserving CPT, while the latter are nonminimal and violate CPT. In section 2, we establish the framework for the parton-model description of factorization in the presence of Lorentz and CPT violation. The application in the context of DIS is presented in section 3. We demonstrate the compatibility of our factorization technique with the operator-product expansion (OPE) and with the Ward identities, and we obtain explicit results for the DIS cross section. In the quark sector, nonzero spin-independent Lorentz-violating operators of mass dimension four are controlled by $c$-type coefficients, while those of dimension five are governed by $a^{(5)}$-type ones. Sensitivities to these coefficients in existing and forthcoming DIS experiments at HERA and the EIC are estimated. In section 4, we investigate factorization in the DY process. The cross sections for nonzero $c$ - and $a^{(5)}$-type coefficients are derived, and attainable sensitivities from experiments at the LHC are estimated. A comparison of our DIS and DY results is performed, revealing the complementary nature of searches at lepton-hadron and hadron-hadron colliders.

Our efforts here to explore spin-independent SME effects in the quark sector extend those in the literature, including studies of single and pair production of $t$ quarks at Fermi National Accelerator Laboratory (Fermilab) and at the LHC [22-24], applications of chiral perturbation theory [25-29], estimates of attainable sensitivities from DIS [30-32], and related investigations [33, 34]. Spin-independent SME coefficients for CPT violation in the quark sector can also be constrained using neutral-meson interferometry [35, 36] via oscillations of kaons [37-41] and of $D, B_{d}$, and $B_{s}$ mesons [42-48]. For $d=5$, these SME coefficients can trigger phenomenologically viable baryogenesis in thermal equilibrium [49-52], thereby avoiding the Sakharov condition of nonequilibrium processes [53]. Cosmic-ray observations imply a few additional bounds on ultrarelativistic combinations of quark-sector coefficients [54]. Other constraints on $d=5$ spin-independent CPT violation have been extracted from experiments with neutrinos, charged leptons, and nucleons [54-60]. 


\section{Framework}

In this section, we present the general procedure for factorization of the scattering cross section in the presence of quark-sector Lorentz violation. To extract the corresponding parton model, we restrict attention to the dominant physical effects occurring at tree level in the electroweak couplings and at zeroth order in the strong coupling.

In the conventional Lorentz-invariant scenario, the parton-model picture of high-energy hadronic processes at large momentum transfer [4] can be shown to emerge from a fieldtheoretic setting under suitable kinematical approximations [61]. For many hadronic processes including DIS and the DY process, each channel contributing to the scattering cross section factorizes into a high-energy perturbative part and a low-energy nonperturbative part, with the latter described by PDFs and fragmentation functions of the hadronic spectators. The perturbative component is often called hard due to the large associated momentum transfer, while the nonperturbative component is called soft. The PDFs are universal in the sense that they are process independent. This factorization becomes most transparent in reference frames in which the dominant momentum regions of the perturbative subprocesses are approximately known. In these frames, asymptotic freedom and the large momentum transfer imply that internal interactions of the hadron constituents occur on a timescale much longer than that of the external probe. The participating constituents may then be treated as freely propagating states. The parton-model picture of scattering emerges by imposing the conservative kinematical restriction to massless and on-shell constituents with momenta collinear to the associated hadrons. For a hadron $H$ with momentum $p^{\mu}=\left(p^{+}, p^{-}, p_{\perp}\right)$ and mass $M$ in lightcone coordinates $p^{ \pm} \equiv \frac{1}{\sqrt{2}}\left(p^{0} \pm p^{3}\right)$ with $p_{\perp} \equiv\left(p^{1}, p^{2}\right)$, a boost from its rest frame along the 3 axis produces a momentum $p^{\mu}=\left(p^{+}, M^{2} / 2 p^{+}, 0_{\perp}\right)$. A constituent of the hadron in the hadron rest frame has a momentum $k$ that scales at most as $k^{\mu} \sim(M, M, M)$. Under a large boost, the constituent inherits the large + momentum because $k^{\mu} \sim\left(p^{+}, M^{2} / p^{+}, M\right)$ up to $\mathcal{O}\left(M / p^{+}\right)$corrections. The ratio $\xi=k^{+} / p^{+}$is boost invariant along the 3 axis and leads to the familiar scaling parametrization $k^{\mu}=\xi p^{\mu}$ of the parton momentum in the massless limit, which is a covariant expression valid in any frame. Scaling permits kinematical approximations that greatly simplify the calculation of the hadronic vertex contribution to the scattering amplitude, and it is known to hold in a wide variety of hadronic processes [62].

In the presence of Lorentz violation, the above perspective requires modification [30]. We focus here on Lorentz-violating operators of arbitrary mass dimension that affect the free propagation of the internal fermion degrees of freedom, including both CPT-even and CPT-odd terms. For simplicity, we disregard possible flavor-changing couplings and limit attention to spin-independent effects. Given the existing tight experimental constraints [11], we can disregard Lorentz and CPT violation in the behavior of the parent hadron itself [30]. The Lorentz- and CPT-violating parton model presented below implements this feature by construction.

For a single massless Dirac fermion, the corresponding gauge-invariant Lorentz- and CPT-violating Lagrange density $\mathcal{L}_{\psi}$ can be written in the form $[32,54]$

$$
\mathcal{L}_{\psi}=\frac{1}{2} \bar{\psi}\left(\gamma^{\mu} i D_{\mu}+\widehat{\mathcal{Q}}\right) \psi+\text { h.c. },
$$


where $D_{\mu}$ is the usual covariant derivative and the operator $\widehat{\mathcal{Q}}$ describes both Lorentzinvariant and Lorentz-violating effects. The explicit form of $\mathcal{L}_{\psi}$ for $d \leq 6$ relevant for our purposes is contained in table I of ref. [32]. The corresponding coefficients for Lorentz violation may be assumed perturbatively small based on current experimental results [11] and the restriction to observer concordant frames [63]. In an inertial frame in the neighborhood of the Earth, all coefficients for Lorentz violation may be taken as spacetime constants, which maintains the conservation of energy and momentum [15]. Field redefinitions and coordinate choices can be used to simplify $\widehat{\mathcal{Q}}$, which reduces the number of coefficients controlling observable effects $[16,17,63-69]$. In this work, we present specific calculations for the coefficients $c_{f}^{\mu \nu}$ at $d=4$ and $a_{f}^{(5) \lambda \mu \nu}$ at $d=5$, where $f=u, d$ spans the two nucleon valence-quark flavors. Other terms in table I of ref. [32] involving coefficients of the $a$ type include $a_{f}^{\mu}$ at $d=3$ and $a_{\mathrm{F} f}^{(5) \lambda \mu \nu}$ at $d=5$, but none of these contribute at leading order to the processes studied here. The field redefinitions insure that the coefficients $c_{f}^{\mu \nu}$ and $a_{f}^{(5) \lambda \mu \nu}$ of interest can be taken to be symmetric in any pair of indices and to have vanishing traces, implying 9 independent observable components of the $c$ type and 16 independent observable components of the $a^{(5)}$ type [36, 69]. Following standard usage in the literature, we denote the symmetric traceless parts of these coefficients as $c_{f}^{\mu \nu}$ and $a_{\mathrm{S} f}^{(5) \lambda \mu \nu}$, where [70]

$$
a_{\mathrm{S} f}^{(5) \lambda \mu \nu}=\frac{1}{3} \sum_{(\lambda \mu \nu)}\left(a_{f}^{(5) \lambda \mu \nu}-\frac{1}{6} a_{f}^{(5) \lambda \alpha \beta} \eta_{\alpha \beta} \eta^{\mu \nu}-\frac{1}{3} a_{f}^{(5) \alpha \lambda \beta} \eta_{\alpha \beta} \eta^{\mu \nu}\right) .
$$

At the quantum level, the theory (2.1) leads to Lorentz-violating propagation and interaction. As a consequence, the conventional dispersion relation $k^{2}=0$ for the 4 momentum of the hadron constituent is modified. The modified dispersion relation can be derived from the Dirac equation (2.1) by setting to zero the strong and electroweak couplings, converting to momentum space, and imposing the vanishing of the determinant of the matrix operator [54]. For the scenarios of interest here, the result can be written in the elegant form

$$
\widetilde{k}^{2}=0
$$

where $\widetilde{k}_{\mu}$ is the Fourier transform of the modified interaction-free Dirac operator. The hadron constituents then propagate along trajectories that are geodesics in a pseudo-Finsler geometry [71-75]. Unlike the Lorentz-invariant case, the modified dispersion relation (2.3) typically involves a non-quadratic relationship between energy and 3-momentum controlled by the coefficients for Lorentz violation. This feature prevents a straightforward identification of the lightcone components of $k$ and complicates attempts at factorization of hadronic processes. An additional challenge arises for the hadron constituents in the initial state during the time of interaction because a momentum parametrization in terms of external kinematics is desired. These points imply that $k$ is no longer the momentum relevant for scaling in a Lorentz-violating parton model, as the relation $k=\xi p$ is no longer consistent with eq. (2.3). Instead, the momentum $\widetilde{k}$ plays the role of interest.

To establish the parton model in the presence of Lorentz violation, we aim to determine the lightcone decomposition of the momentum $\widetilde{k}$ of an on-shell massless quark, which is 
subject to the condition (2.3). The perturbative nature of Lorentz violation implies that the frame appropriate for factorization differs at most from conventional frame choices by an $\mathcal{O}(\widehat{\mathcal{Q}})$ transformation. Since a large portion of the space of SME coefficients for nucleons is strongly constrained by experiment [11], we can reasonably neglect Lorentz-violating effects in the initial- and final-state hadrons. We therefore seek a frame in which $\widetilde{k}$ can be parametrized in terms of its parent hadron momentum $p$ and the parton coefficients for Lorentz violation in $\widehat{\mathcal{Q}}$. To retain the equivalent on-shell condition in a covariant manner, we choose

$$
\widetilde{k}^{\mu}=\xi p^{\mu} .
$$

Since the effects of Lorentz violation are perturbative, one may still argue that $\widetilde{k}^{\mu} \sim(M, M, M)$ in the rest frame of the hadron. Performing an observer boost along the 3 axis yields $\widetilde{k} \sim\left(p^{+}, M^{2} / p^{+}, M\right)$, where now the variable $\xi \equiv \widetilde{k}^{+} / p^{+}$plays the role of the parton momentum fraction. Note that the frame changes implemented by the observer boosts are accompanied by covariant transformations of the coefficients for Lorentz violation [15]. The desired procedure is therefore to impose the conditions (2.3)-(2.4) and perform the factorization of the hadronic scattering amplitude working in an appropriate observer frame from which the calculation can proceed in parallel with the conventional case.

The momentum $\widetilde{k}_{\mu}$ is defined for a parton via eq. (2.1). However, other internal momenta appear in the scattering process. In DIS, for example, the initial parton momentum $k$ differs from the final parton momentum $k+q$ by the momentum $q$ of the vector boson. For calculational purposes, it is convenient to introduce a momentum $\widetilde{q}$ defined as the difference of the modified momenta for the final and initial partons,

$$
\widetilde{q} \equiv \widetilde{k+q}-\widetilde{k} .
$$

In the presence of Lorentz violation involving operators of dimensions $d=3$ and 4 , the explicit form of $\widetilde{q}$ can be written in terms of $q$ and SME coefficients, independent of $k$. For $d>4$, however, the definition (2.5) implies that $\widetilde{q}$ depends nontrivially on $k$ as well, which complicates the derivation of the cross section. In this work, we explore the implications of both these types of situations for DIS and the DY process.

\section{Deep inelastic scattering}

In this section, we apply the general procedure outlined in section 2 to inclusive leptonhadron DIS. The special case of unpolarized electron-proton DIS mediated by conventional photon and $Z^{0}$ exchange in the presence of minimal quark-sector Lorentz violation has been studied and applied in the context of HERA data [30] and the future EIC [31]. Analogous results for nonminimal Lorentz and CPT violation have also been obtained [32]. Here, we show how these results fit within the new formalism and provide both updated and new numerical estimates of attainable sensitivities to Lorentz violation. Effects on DIS of minimal Lorentz violation in the weak sector are considered in ref. [34].

\subsection{Factorization of the hadronic tensor}

The inclusive DIS process $l+H \rightarrow l^{\prime}+X$ describes a lepton $l$ scattering on a hadron $H$ into a final-state lepton $l^{\prime}$ and an unmeasured hadronic state $X$. The interaction is mediated by 
a spacelike boson of momentum $q=l-l^{\prime}$. It is convenient to introduce the dimensionless Bjorken variables

$$
x=\frac{-q^{2}}{2 p \cdot q}, \quad y=\frac{p \cdot q}{p \cdot l},
$$

where $p$ is the hadron momentum. The DIS limit is characterized by $-q^{2} \equiv Q^{2} \rightarrow \infty$ with $x$ fixed. This produces a final-state invariant mass much larger than the hadron mass $M$, which may therefore be neglected. Reviews of DIS and related processes include refs. [76, 77].

The observable of interest is the differential cross section $d \sigma$, which by its conventional definition is a Lorentz-scalar quantity built from the invariant amplitude, an initial-state flux factor, and a contribution from the final-state phase space. In principle, Lorentz violation could affect each of these, so care is required in calculating the cross section [78]. In this work, Lorentz violation can enter only through the hadronic portion of the full scattering amplitude because the exchanged vector boson, the incoming particle flux, and the phase space of the outgoing particles are assumed conventional. The cross section as a function of the lepton phase-space variables $x, y$ and $\phi$ takes the form

$$
\frac{d \sigma}{d x d y d \phi}=\frac{\alpha^{2} y}{2 \pi Q^{4}} \sum_{i} R_{i}\left(L_{i}\right)_{\mu \nu}\left(\operatorname{Im} T_{i}\right)^{\mu \nu} .
$$

In this expression, the index $i$ denotes the neutral-current channels $i=\gamma, Z$ or the chargedcurrent channel $i=W^{ \pm}$, with corresponding lepton tensor $\left(L_{i}\right)_{\mu \nu}$ and forward amplitude $\left(T_{i}\right)^{\mu \nu}$. The factor $R_{i}$ denotes the ratio of the exchanged boson propagator to the photon propagator. Unitarity has been used to write the hadronic tensor $\left(W_{i}\right)^{\mu \nu}$ in terms of the imaginary part of its forward amplitude $\left(\operatorname{Im} T_{i}\right)^{\mu \nu}$ via the optical theorem in the physical scattering region $q^{2}<0$. This operation remains valid in the SME context since all potential new effects are associated with hermitian operators [79]. The forward amplitude is defined as

$$
T_{\mu \nu}=i \int d^{4} w e^{i q \cdot w}\left\langle p, s\left|\mathrm{~T} j_{\mu}^{\dagger}(w) j_{\nu}(0)\right| p, s\right\rangle_{c},
$$

where $\mathrm{T} j_{\mu}^{\dagger}(w) j_{\nu}(0)$ is the time-ordered product of electroweak quark currents $j_{\mu}^{\dagger}(w), j_{\nu}(0)$. The hadron spin vector $s^{\mu}$ satisfies $s^{2}=-M^{2}, s \cdot p=0$, and $c$ denotes the restriction to connected matrix elements. For simplicity, we suppress the subscript $c$, the channel label $i$, and possible flavor labels in the following discussion.

Given that eq. (2.1) in principle contains higher-order derivative terms, the generalized Euler-Lagrange equations must be used to derive the global $\mathrm{SU}(N)$ current $j^{\mu}$. Note that only terms with $d \geq 4$ augment the current from its conventional form. We denote the general Dirac structure of these contributions to be $\Gamma^{\mu}$ and for simplicity write the current as

$$
j_{\psi \chi}^{\mu}=: \bar{\psi} \Gamma^{\mu} \chi
$$

where typically $\psi \neq \chi$ and the associated charges are implicit. In the DIS limit, asymptotic freedom implies that the first-order electroweak interaction provides the dominant contribution to the hadronic portion of the scattering amplitude. We therefore evaluate eq. (3.3) at zeroth order in the strong-interaction coupling, giving

$$
T^{\mu \nu}=i \int d^{4} w e^{i q \cdot w}\left\langle p, s\left|: \bar{\psi}(w) \Gamma^{\mu} i S_{F}(w) \Gamma^{\nu} \psi(0):+: \bar{\psi}(0) \Gamma^{\nu} i S_{F}(-w) \Gamma^{\mu} \psi(w):\right| p, s\right\rangle,
$$


with the Feynman propagator

$$
i S_{F}(x-y)=i \int_{C_{F}} \frac{d^{4} k}{(2 \pi)^{4}} \frac{e^{-i k \cdot(x-y)}}{\widetilde{k}+i \epsilon},
$$

where $\widetilde{k} \equiv \gamma_{\alpha} \widetilde{k}^{\alpha}$.

Unlike the conventional case, the structure $\Gamma^{\mu} S_{F} \Gamma^{\nu}$ can contain both even and odd powers of gamma matrices, which leads to additional contributions. Each term in eq. (3.5) can be viewed as a matrix $X$ and can be expanded in a basis $\Gamma^{A}$ of gamma matrices as $X=x_{A} \Gamma^{A}$. The conventional completeness relation $\operatorname{Tr}\left[\Gamma^{A} \Gamma_{B}\right]=4 \delta^{A}{ }_{B}$ implies $x_{A}=(1 / 4) \operatorname{Tr}\left[\Gamma_{A} X\right]$. To match with the results common in the literature, we choose the basis

$$
\begin{aligned}
& \Gamma_{A}=\left\{\mathbb{1}, \gamma_{5}, \gamma_{\mu}, \gamma_{5} \gamma_{\mu}, i \gamma_{5} \sigma_{\mu \nu}\right\}, \\
& \Gamma^{A}=\left\{\mathbb{1}, \gamma_{5}, \gamma^{\mu}, \gamma^{\mu} \gamma_{5},-i \gamma_{5} \sigma^{\mu \nu} / 2\right\}
\end{aligned}
$$

With Dirac indices explicitly displayed, one has

$$
: \psi_{a}(0) \bar{\psi}_{b}(x):=-\frac{1}{4} \bar{\psi}(x) \Gamma_{A} \psi(0)\left(\Gamma^{A}\right)_{a b},
$$

giving

$$
\begin{aligned}
& T^{\mu \nu}=-\frac{1}{4} \int \frac{d^{4} k}{(2 \pi)^{4}}\left(\operatorname{Tr}\left[\Gamma^{\mu} \frac{1}{\gamma_{\alpha} \widetilde{k+q}^{\alpha}+i \epsilon} \Gamma^{\nu}\right] \int d^{4} w e^{-i k \cdot w}\langle p, s|\bar{\psi}(w) \psi(0)| p, s\rangle\right. \\
& +\operatorname{Tr}\left[\Gamma^{\mu} \frac{1}{\gamma_{\alpha} \widetilde{k+q}^{\alpha}+i \epsilon} \Gamma^{\nu} \gamma_{5}\right] \int d^{4} w e^{-i k \cdot w}\left\langle p, s\left|\bar{\psi}(w) \gamma_{5} \psi(0)\right| p, s\right\rangle \\
& +\operatorname{Tr}\left[\Gamma^{\mu} \frac{1}{\gamma_{\alpha} \widetilde{k+q}^{\alpha}+i \epsilon} \Gamma^{\nu} \gamma^{\rho}\right] \int d^{4} w e^{-i k \cdot w}\left\langle p, s\left|\bar{\psi}(w) \gamma_{\rho} \psi(0)\right| p, s\right\rangle \\
& +\operatorname{Tr}\left[\Gamma^{\mu} \frac{1}{\gamma_{\alpha} \widetilde{k+q}^{\alpha}+i \epsilon} \Gamma^{\nu} \gamma^{\rho} \gamma_{5}\right] \int d^{4} w e^{-i k \cdot w}\left\langle p, s\left|\bar{\psi}(w) \gamma_{5} \gamma_{\rho} \psi(0)\right| p, s\right\rangle \\
& -\frac{1}{2} \operatorname{Tr}\left[\Gamma^{\mu} \frac{1}{\gamma_{\alpha} \widetilde{k+q}^{\alpha}+i \epsilon} \Gamma^{\nu} i \gamma_{5} \sigma^{\rho \sigma}\right] \int d^{4} w e^{-i k \cdot w}\left\langle p, s\left|\bar{\psi}(w) i \gamma_{5} \sigma_{\rho \sigma} \psi(0)\right| p, s\right\rangle \\
& +(q \leftrightarrow-q, 0 \leftrightarrow w, \mu \leftrightarrow \nu)) .
\end{aligned}
$$

Note that normal ordering of operators is implied.

Taking the imaginary part of $T^{\mu \nu}$, the terms that depend on $k+q$ or $k-q$ contribute only via scattering initiated by a quark or antiquark, respectively. The imaginary part of $T^{\mu \nu}$ comes solely from the propagator denominators because the combination of spatial integration, exponential factors, and matrix-element terms is hermitian. This feature is a consequence of translation invariance, which remains a symmetry within the SME framework when the coefficients for Lorentz violation are spacetime constants. The imaginary 
piece of the propagator takes the form

$$
\operatorname{Im}\left(\frac{1}{\widetilde{k}^{2}+i \epsilon}\right)=-\pi \delta\left(\widetilde{k}^{2}\right) \theta\left(k^{0}\right)-\pi \delta\left(\widetilde{-k}^{2}\right) \theta\left(-k^{0}\right),
$$

where the two terms correspond to particle and antiparticle. For coefficients controlling CPT-even effects one finds $\widetilde{-k}=-\widetilde{k}$, implying the particle and antiparticle have the same dispersion relation. For coefficients governing CPT violation, $\widetilde{k}$ lacks a definite parity in $k$, implying the particle and antiparticle have different dispersion relations that are related by changing the signs of the coefficients for CPT violation. In what follows we focus on the quark contribution, so $\widetilde{k}$ is calculated with the sign corresponding to a particle. Moreover, in applying the standard Cutkosky rules, the intermediate propagator in the diagram with an incoming quark uniquely forces the dispersion relation for the intermediate quark to be identical to the incoming quark one, so that $\widetilde{k}^{2}=(\widetilde{k+q})^{2}=0$.

The relevant kinematics can be handled by working in lightcone coordinates and in the Breit frame, which in the conventional case is defined as the center-of-mass (CM) frame of the hadron and exchanged boson, $\vec{p}+\vec{q}=\overrightarrow{0}$. In light of eq. (2.5), however, we must here introduce a modified Breit frame defined by the relation $\vec{p}+\overrightarrow{\widetilde{q}}=\overrightarrow{0}$. The hadron and shifted virtual boson kinematics may be parametrized as

$$
\begin{aligned}
p^{\mu} & =\left(p^{+}, \frac{M^{2}}{2 p^{+}}, 0_{\perp}\right), \\
\widetilde{q}^{\mu} & =\left(-\widetilde{x} p^{+}, \frac{\widetilde{Q}^{2}}{2 \widetilde{x} p^{+}}, 0_{\perp}\right),
\end{aligned}
$$

where

$$
\widetilde{x}=\frac{-\widetilde{q}^{2}}{2 p \cdot \widetilde{q}}
$$

with $-\widetilde{q}^{2} \equiv \widetilde{Q}^{2}$. In writing eq. (3.11), we neglect corrections of order $\mathcal{O}\left(M^{2} / Q\right)$ and the zeroth component $\widetilde{q}^{0}$ with respect to $\widetilde{Q}$. Note also that $\widetilde{q}$ differs from the physical boson momentum $q$ only if operators with $d \geq 4$ are taken into consideration, so the modified Breit frame differs from the conventional one only in the presence of these operators. Consideration of eq. (2.5) implies that $\widetilde{q}$ and hence $\widetilde{x}$ are functions of $k, q$, and the coefficients for Lorentz violation. Therefore, for nonminimal interactions the modified Breit frame depends on a polynomial in $\xi$. However, since additional dependence on powers of $\xi$ is accompanied by coefficients for Lorentz violation, the replacement $\xi \rightarrow x$ holds at leading order in Lorentz violation and so both $\widetilde{q}$ and $\widetilde{x}$ can be constructed event by event from the incident hadron and scattered lepton kinematics. Based on the discussion in section 2, we can parametrize the large + component of $\widetilde{k}^{+}$as $\xi p^{+}$with virtualities $\widetilde{k}^{-} \sim M^{2} / p^{+}$and $\widetilde{k}_{\perp} \sim M$. This yields

$$
\begin{aligned}
\widetilde{k}^{\mu} & =\left(\xi p^{+}, \widetilde{k}^{-}, \widetilde{k}_{\perp}\right) \\
\widetilde{k}^{\prime \mu} & =\left((\xi-\widetilde{x}) p^{+}, \frac{\widetilde{Q}^{2}}{2 \widetilde{x} p^{+}}+\widetilde{k}^{-}, \widetilde{k}_{\perp}\right),
\end{aligned}
$$


where $\widetilde{k}^{\mu}=\widetilde{k+q}^{\mu}$. The structure of these equations and of eqs. (3.11)-(3.12) is standard but involves replacing conventional variables with tilde ones. In the usual scenario $\xi$ and $x$ differ by corrections of $\mathcal{O}\left(M^{2} / Q^{2}\right)$, implying the scaling $k^{\prime+} \sim M^{2} / p^{+}, k^{\prime-} \sim p^{+}$, so the boson transfers the incident parton from the + to - lightcone direction. In the present case, the dominance of the - component of $\widetilde{k^{\prime}}$ over the + component still persists because corrections from Lorentz-violating effects are suppressed relative to $p^{+} \sim Q$.

Proceeding with the spatial and momentum integrations in $T^{\mu \nu}$ requires a change of variables $k \rightarrow \widetilde{k}$ because only the latter momentum exhibits the scaling of interest. To evaluate the $w$ integration in a straightforward way, a transformation $w \rightarrow \widehat{w}$ must be performed such that $k \cdot w=\widetilde{k} \cdot \widehat{w}$. Neglecting the small components of $\widetilde{k}$ with respect to the large + and - components of $\widetilde{q}$, one finds that $\widetilde{k}^{-}$and $\widetilde{k}_{\perp}$ can be disregarded in the hard scattering up to corrections of $\mathcal{O}(M / Q)$. This is the analogue in the modified Breit frame of the conventional result. The integrations over $\widetilde{k}^{-}$and $\widetilde{k}_{\perp}$ thus bypass the traces, and the structures in the traces proportional to $\gamma^{-}, \gamma^{-} \gamma_{5}, \gamma^{-} \gamma_{\perp}^{i} \gamma_{5}$ provide the dominant contributions to $T^{\mu \nu}$ for a hadron with a large + momentum and so are accompanied by large + components in the hadronic matrix elements. It is thus reasonable to take $\gamma^{\rho} \approx \gamma^{-}$in the traces and $\gamma_{\rho} \approx \gamma^{+}$in the matrix elements. Bearing these considerations and eq. (3.13) in mind, we obtain

$$
\begin{aligned}
T_{f}^{\mu \nu} \simeq \int \frac{d \widetilde{k}^{+}}{\widetilde{k}^{+}} \operatorname{Tr} & {\left[\Gamma^{\mu} \frac{-1}{\gamma_{\alpha} \widetilde{k+q}^{\alpha}+i \epsilon} \Gamma^{\nu} \frac{\widetilde{k}}{2}\right.} \\
& \left.\times\left(\mathbb{1} f_{f}\left(\widetilde{k}^{+}\right)-\gamma_{5} \lambda \Delta f_{f}\left(\widetilde{k}^{+}\right)+\gamma_{5} \gamma_{\perp}^{i} \lambda_{\perp} \Delta_{\perp} f_{f}\left(\widetilde{k}^{+}\right)\right)\right],
\end{aligned}
$$

where we have neglected diagrams proportional to $1 /\left(\gamma_{\alpha} \widetilde{k-q}+i \epsilon\right)$ because they vanish in the physical scattering region. The unintegrated PDFs here are defined as

$$
\begin{gathered}
f_{f}\left(\widetilde{k}^{+}, \ldots\right) \equiv \int \frac{d \widetilde{k}^{-} d \widetilde{k}_{\perp} d^{4} \widehat{w}}{(2 \pi)^{4}} J_{k} J_{w} e^{-i \widetilde{k} \cdot \widehat{w}}\left\langle p, s\left|\bar{\psi}_{f}(w(\widehat{w})) \frac{\gamma^{+}}{2} \psi_{f}(0)\right| p, s\right\rangle, \\
\lambda \Delta f_{f}\left(\widetilde{k}^{+}, \ldots\right) \equiv \int \frac{d \widetilde{k}^{-} d \widetilde{k}_{\perp} d^{4} \widehat{w}}{(2 \pi)^{4}} J_{k} J_{w} e^{-i \widetilde{k} \cdot \widehat{w}}\left\langle p, s\left|\bar{\psi}_{f}(w(\widehat{w})) \frac{\gamma^{+} \gamma_{5}}{2} \psi_{f}(0)\right| p, s\right\rangle, \\
\lambda_{\perp} \Delta_{\perp} f_{f}\left(\widetilde{k}^{+}, \ldots\right) \equiv \int \frac{d \widetilde{k}^{-} d \widetilde{k}_{\perp} d^{4} \widehat{w}}{(2 \pi)^{4}} J_{k} J_{w} e^{-i \widetilde{k} \cdot \widehat{w}}\left\langle p, s\left|\bar{\psi}_{f}(w(\widehat{w})) \frac{\gamma^{+} \gamma_{\perp}^{i} \gamma_{5}}{4} \psi_{f}(0)\right| p, s\right\rangle,
\end{gathered}
$$

where $\lambda, \lambda_{\perp}$ are the longitudinal and transverse target helicities and $\Delta f_{f}, \Delta_{\perp} f_{f}$ are the corresponding longitudinal and transverse polarized PDFs. We have also introduced the lightcone definitions of the gamma matrices, $\gamma^{ \pm}=\frac{1}{\sqrt{2}}\left(\gamma^{0}+\gamma^{3}\right), \gamma_{\perp}^{i}=\gamma^{1}, \gamma^{2}$. The ellipses in the arguments on the left-hand side of eq. (3.15) denote possible dependences on the coefficients for Lorentz violation. The factors $J_{k}, J_{w}$ are jacobians from the change of variables, which differ from unity at first order in Lorentz violation. These expressions represent the modified dominant twist-two PDFs. They differ from conventional results by the jacobians and by the dependences on $w(\widehat{w})$ in the matrix elements.

In the limit of vanishing coefficients for Lorentz violation, we have $J_{k}=J_{w}=1$, $\widetilde{k} \rightarrow k, \widehat{w} \rightarrow w$, and the PDFs reduce to functions of a single variable that can be expressed 
covariantly in terms of two light-like vectors

$$
\bar{n}^{\mu}=\frac{1}{\sqrt{2}}(1,0,0,+1), \quad n^{\mu}=\frac{1}{\sqrt{2}}(1,0,0,-1),
$$

with $n^{2}=\bar{n}^{2}=0, n \cdot \bar{n}=1$. In this basis, a generic four-vector $A^{\mu}$ can be expanded as

$$
A^{\mu}=(n \cdot A) \bar{n}^{\mu}+(\bar{n} \cdot A) n^{\mu}+A_{\perp}^{\mu},
$$

with $A^{+}=n \cdot A, A^{-}=\bar{n} \cdot A$. We employ the basis (3.16) and parametrize $w=\lambda n$ with $\lambda$ a positive constant. Since scaling $n$ by a positive constant implies scaling $\lambda$ oppositely, the PDFs are be invariant under scaling of $n$. The only scalar combination allowed is $k \cdot n / p \cdot n=\xi$, so the PDFs can depend only on $\xi$. Performing the $k^{-}$and $\vec{k}_{\perp}$ integrations produces delta functions that set $w^{+}=\vec{w}_{\perp}=0$, which yields the standard result with PDFs as matrix elements of bilocal operators on the lightcone,

$$
\begin{aligned}
f_{f}(\xi) & =\int \frac{d \lambda}{2 \pi} e^{-i \xi p \cdot n \lambda}\left\langle p\left|\bar{\psi}_{f}(\lambda n) \frac{\not h}{2} \psi_{f}(0)\right| p\right\rangle, \\
\lambda \Delta f_{f}(\xi) & =\int \frac{d \lambda}{2 \pi} e^{-i \xi p \cdot n \lambda}\left\langle p, s\left|\bar{\psi}_{f}(\lambda n) \frac{\not h \gamma_{5}}{2} \psi_{f}(0)\right| p, s\right\rangle, \\
\lambda_{\perp} \Delta_{\perp} f_{f}(\xi) & =\int \frac{d \lambda}{2 \pi} e^{-i \xi p \cdot n \lambda}\left\langle p, s\left|\bar{\psi}_{f}(\lambda n) \frac{\not h \gamma_{\perp}^{i} \gamma_{5}}{4} \psi_{f}(0)\right| p, s\right\rangle .
\end{aligned}
$$

Note that the rotational properties of the quark bilinear appearing in $f_{f}(\xi)$ imply this $\mathrm{PDF}$ is independent of the hadron spin $s$. In the presence of nonvanishing coefficients for Lorentz violation, the situation is more complicated. Explicit expressions at the level of eq. (3.18) can be deduced by a similar procedure and yield scalar functions, but these are in general somewhat involved. As shown in section 3.2, the PDFs acquire additional dependence on the complete contraction of the coefficients for Lorentz violation with the hadron momentum.

Taking the imaginary part of eq. (3.14) by using eq. (3.10) and integrating over the longitudinal variable sets $\xi$ to a function of $x, p, q$, and the coefficients for Lorentz violation. The resulting form of $T^{\mu \nu}$ is factorized and depicted in figure 1. We have thus demonstrated that working in the modified Breit frame $\vec{p}+\widetilde{\vec{q}}=\overrightarrow{0}$ defined by eq. (3.11) leads to factorization of $T^{\mu \nu}$. As in the conventional case, the PDFs in eq. (3.15) emerge as nonlocal matrix elements evaluated along the + lightcone direction. Since the PDFs remain scalar quantities and the perturbative portion of $T^{\mu \nu}$ is a covariant expression in the external momenta, the definition of the PDFs, the momentum fraction, and the cross section hold in any frame. Contraction with the lepton tensor $\left(L_{i}\right)^{\mu \nu}$ in the channels of interest and combining the result with the additional kinematical factors then yields the scattering cross section.

\subsection{The operator product expansion}

The hadronic tensor $W^{\mu \nu}$ and the forward amplitude $T^{\mu \nu}$ can also be calculated using the OPE approach [30]. In this section, we sharpen our discussion by generalizing previous results and connecting to the PDFs in eq. (3.15). The OPE considers the expansion of the product of spacelike-separated operators, such as the product of hadronic currents that 


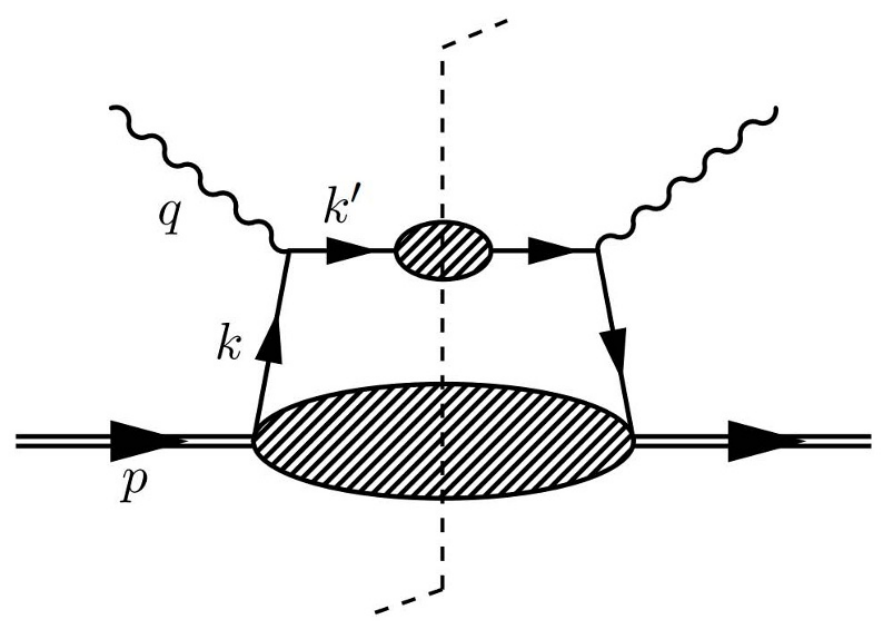

Figure 1. Depiction of the dominant contributions to the hadronic tensor of the DIS process. The upper portion of the graph represents the tree-level perturbative process with final-state interactions denoted by the small hatched ellipse, which can be neglected in practice. The larger hatched ellipse denotes the effective long-distance physics encapsulated by the PDFs. The dashed line bisecting the diagram indicates summation over final hadronic states and placing intermediate states on mass shell, $\widetilde{k^{\prime}}=0$.

frequently appears in scattering processes, as a sum of local operators in the short-distance limit. Note that the short-distance expansion of the currents occurs outside of the physical scattering region.

For minimal $c$-type coefficients, a direct evaluation of the current product [30] yields operators of the form $\bar{\psi}_{f}(0) \gamma^{\mu_{1}}\left(i \widetilde{\partial}^{\mu_{2}}\right)\left(i \widetilde{\partial}^{\mu_{3}}\right) \ldots\left(i \widetilde{\partial}^{\mu_{n}}\right) \psi_{f}(0)$. The calculation of the hadronic tensor requires matrix elements of these operators between hadron states. Taking tree-level matrix elements of these operators between quark states of momentum $k$ gives

$$
\left\langle k\left|\bar{\psi}_{f} \gamma^{\mu_{1}} i \widetilde{\partial}^{\mu_{2}} \cdots i \widetilde{\partial}^{\mu_{n}} \psi_{f}\right| k\right\rangle \propto \widetilde{k}^{\mu_{1}} \ldots \widetilde{k}^{\mu_{n}}
$$

which is totally symmetric and traceless because $\widetilde{k}^{2}=0$. This suggests that only the symmetric and traceless parts of the operators

$$
\mathcal{O}_{f}^{\mu_{1} \cdots \mu_{n}}=\bar{\psi}_{f} \gamma^{\left\{\mu_{1}\right.}\left(i \widetilde{D}^{\mu_{2}}\right)\left(i \widetilde{D}^{\mu_{3}}\right) \ldots\left(i \widetilde{D}^{\left.\mu_{n}\right\}}\right) \psi_{f}-\text { traces }
$$

enter at leading twist, where $\widetilde{D}^{\mu}$ represents the covariant extension of $\widetilde{\partial}^{\mu}$. Moreover, the factorization analysis implies that the partons in the hard scattering have momentum $k^{\mu}$ such that $\widetilde{k}^{\mu} \propto p^{\mu}$, thus suggesting

$$
\left\langle p\left|\mathcal{O}_{f}^{\mu_{1} \cdots \mu_{n}}\right| p\right\rangle=2 \mathcal{A}_{n}^{f} p^{\mu_{1}} \cdots p^{\mu_{n}},
$$

where the quantities $\mathcal{A}_{n}^{f}$ typically depend on $Q$ and possibly on scalar contractions of the hadron momentum with the coefficients for Lorentz violation. For $n=2$, this result is supported directly by noting that

$$
\mathcal{O}_{f}^{\mu_{1} \mu_{2}}=\theta_{f \alpha \beta}\left(\eta^{\alpha \mu_{1}} \eta^{\beta \mu_{2}}+\eta^{\alpha \mu_{2}} \eta^{\beta \mu_{1}}\right)-\text { traces, }
$$


where $\theta_{f}^{\mu \nu}$ is the symmetric part of the energy-momentum tensor, and hence that

$$
\left\langle p\left|\mathcal{O}_{f}^{\mu_{1} \mu_{2}}\right| p\right\rangle=\left\langle p\left|\theta_{f \alpha \beta}\right| p\right\rangle\left(\eta^{\alpha \mu_{1}} \eta^{\beta \mu_{2}}+\eta^{\alpha \mu_{2}} \eta^{\beta \mu_{1}}\right)-\operatorname{traces} \propto p^{\mu_{1}} p^{\mu_{2}},
$$

implying that $\mathcal{A}_{2}^{f}$ is the fraction of the total energy-momentum of the hadron carried by the parton.

Given the form of eq. (3.21), the prediction for the DIS cross section is identical to the factorization result if the matrix elements $\mathcal{A}_{n}^{f}$ yield the moments of the PDFs,

$$
\int d \widetilde{k}^{+}\left(\widetilde{k}^{+}\right)^{n} f_{f}\left(\widetilde{k}^{+}\right)=(n \cdot p)^{n+1} \mathcal{A}_{n+1}^{f} .
$$

To show that this indeed holds, consider the slightly more general case of coefficients for Lorentz violation $A^{\mu_{1} \cdots \mu_{m+1}}$ with $m+1$ indices, for which we have

$$
\begin{aligned}
f_{f}\left(\widetilde{k}^{+}\right) & =\int \frac{d \widetilde{k}^{-} d \widetilde{k}_{\perp} d^{4} \widehat{w}}{(2 \pi)^{4}} J_{k} J_{w} e^{-i \widetilde{k} \cdot \widehat{w}}\left\langle p\left|\bar{\psi}_{f}(w(\widehat{w})) \frac{\not h}{2} \psi_{f}(0)\right| p\right\rangle \\
& \equiv \int \frac{d \widetilde{k}^{-} d \widetilde{k}_{\perp} d^{4} \widehat{w}}{(2 \pi)^{4}} J_{k} J_{w} e^{-i \widetilde{k} \cdot \widehat{w}} F(w(\widehat{w})), \\
\widetilde{k}^{\mu} & =k^{\mu}-A^{\mu k \cdots k}, \quad \widehat{w}^{\mu}=w^{\mu}+A^{w \mu k \cdots k}, \\
J_{k} & =1+\left(A^{\mu \nu \widetilde{k} \cdots \widetilde{k}}+A^{\mu \widetilde{k} \nu \widetilde{k} \cdots \widetilde{k}}+\cdots+A^{\mu \widetilde{k} \cdots \widetilde{k} \nu}\right) \eta_{\mu \nu}, \quad J_{w}=1-A^{\mu \nu \widetilde{k} \cdots \widetilde{k}} \eta_{\mu \nu}, \\
w(\widehat{w})^{\mu} & =\widehat{w}^{\mu}-A^{\widehat{w} \mu \widetilde{k} \cdots \widetilde{k}} .
\end{aligned}
$$

The following manipulations allow the removal of the explicit dependence on the jacobians $J_{k, w}$ :

$$
\begin{aligned}
f_{f}\left(\widetilde{k}^{+}\right)= & \int \frac{d \widetilde{k}^{-} d \widetilde{k}_{\perp} d^{4} \widehat{w}}{(2 \pi)^{4}} J_{k} J_{w} e^{-i \widetilde{k} \cdot \widehat{w}} F\left(\widehat{w}-A^{\widehat{w} \mu \widetilde{k} \cdots \widetilde{k}}\right) \\
& \stackrel{(1)}{=} \int \frac{d \widetilde{k}^{-} d \widetilde{k}_{\perp} d^{4} \widehat{w}}{(2 \pi)^{4}} J_{k} J_{w} e^{-i \widetilde{k} \cdot \widehat{w}}\left(1-A^{\widehat{w} \mu \widetilde{k} \cdots \widetilde{k}} \frac{\partial}{\partial \widehat{w}^{\mu}}\right) F(\widehat{w}) \\
& \stackrel{(2)}{=} \int \frac{d \widetilde{k}^{-} d \widetilde{k}_{\perp} d^{4} w}{(2 \pi)^{4}} J_{k} J_{w} e^{-i \widetilde{k} \cdot \widehat{w}}\left(1+A^{\nu \mu \widetilde{k} \cdots \widetilde{k}} \eta_{\mu \nu}-i A^{\widehat{w} \widetilde{k} \cdots \widetilde{k}}\right) F(\widehat{w}) \\
\stackrel{(3)}{=} & \int \frac{d \widetilde{k}^{-} d \widetilde{k}_{\perp} d^{4} \widehat{w}}{(2 \pi)^{4}} J_{k} J_{w} F(\widehat{w})\left(1+A^{\nu \mu \widetilde{k} \cdots \widetilde{k}} \eta_{\mu \nu}+A^{\mu \widetilde{k} \cdots \widetilde{k}} \frac{\partial}{\partial \widetilde{k}^{\mu}}\right) e^{-i \widetilde{k} \cdot \widehat{w}} \\
\stackrel{(4)}{=} & \int \frac{d \widetilde{k}^{-} d \widetilde{k}_{\perp} d^{4} \widehat{w}}{(2 \pi)^{4}} J_{k} J_{w} F(\widehat{w}) e^{-i \widetilde{k} \cdot \widehat{w}}\left(1+A^{\nu \mu \widetilde{k} \cdots \widetilde{k}} \eta_{\mu \nu}\right. \\
& \left.-\left(A^{\mu \nu \widetilde{k} \cdots \widetilde{k}}+A^{\mu \widetilde{k} \nu \widetilde{k} \cdots \widetilde{k}}+\cdots+A^{\mu \widetilde{k} \cdots \widetilde{k} \nu}\right) \eta_{\mu \nu}-A^{\mu \widetilde{k} \cdots \widetilde{k}} \frac{\partial}{\partial \widetilde{k}^{\mu}}\right) \\
= & \int \frac{d \widetilde{k}^{-} d \widetilde{k}_{\perp} d^{4} \widehat{w}}{(2 \pi)^{4}} J_{k} J_{w} F(\widehat{w}) e^{-i \widetilde{k} \cdot \widehat{w}} J_{k}^{-1} J_{w}^{-1}\left(1-A^{\mu \widetilde{k} \cdots \widetilde{k}} \frac{\partial}{\partial \widetilde{k}^{\mu}}\right) \\
= & \int \frac{d \widetilde{k}^{-} d \widetilde{k}_{\perp} d^{4} \widehat{w}}{(2 \pi)^{4}} F(\widehat{w}) e^{-i \widetilde{k} \cdot \widehat{w}}\left(1-A^{\mu \widetilde{k} \cdots \widetilde{k}} \frac{\partial}{\partial \widetilde{k}^{\mu}}\right) \\
\stackrel{(5)}{=} & \int \frac{d \widetilde{k}^{-} d \widetilde{k}_{\perp} d^{4} \widehat{w}}{(2 \pi)^{4}} F(\widehat{w}) e^{-i \widetilde{k} \cdot \widehat{w}}\left(1-A^{\mu \widetilde{k} \cdots \widetilde{k}} n_{\mu} \frac{\partial}{\partial \widetilde{k}^{+}}\right) \cdot
\end{aligned}
$$


In step (1) of this derivation we expanded $F$, in step (2) we integrated by parts in $\widehat{w}$, in step (3) we expressed the term linear in $\widehat{w}$ as a $\widetilde{k}$ derivative acting only on $\exp (-i \widetilde{k} \cdot \widehat{w})$, and in step (4) we integrated by parts in $\widetilde{k}$ noting that $f$ is a distribution that must be integrated over a hard-scattering kernel. Finally, in step (5) we used the fact that the hard scattering is a function of $\widetilde{k}^{+}$alone and that in lightcone coordinates one has

$$
\frac{\partial}{\partial \widetilde{k}^{\mu}}=n_{\mu} \frac{\partial}{\partial \widetilde{k}^{+}}+\bar{n}_{\mu} \frac{\partial}{\partial \widetilde{k}^{-}}+\frac{\partial}{\partial \widetilde{k}_{\perp}^{\mu}}
$$

To proceed further, we observe that the integral over terms proportional to $\widetilde{k^{-a}}$ and $\widetilde{k}_{\perp}^{b}$ with $a, b \geq 1$ produce delta functions $\delta^{(a)}\left(\widehat{w}^{+}\right)$and $\delta^{(b)}\left(\widehat{w}_{\perp}\right)$. After integrating over $\widehat{w}$, these yield higher-twist PDFs that we can neglect as higher order. This implies that we can set $\widetilde{k}^{\mu}=\widetilde{k}^{+} \bar{n}^{\mu}$ in the last term of eq. (3.26), integrate over $\widetilde{k}^{-}, \widetilde{k}_{\perp}, \widehat{w}^{+}$, and $\widehat{w}_{\perp}$, and obtain

$$
\begin{aligned}
f_{f}\left(\widetilde{k}^{+}\right) & =\int \frac{d \widehat{w}^{-}}{2 \pi} F\left(\widehat{w}^{-} n\right) e^{-i \widetilde{k}^{+} \widehat{w}^{-}}\left(1-A^{n \bar{n} \cdots \bar{n}}\left(\widetilde{k}^{+}\right)^{m} \frac{\partial}{\partial \widetilde{k}^{+}}\right) \\
& =\int \frac{d \widehat{w}^{-}}{2 \pi} e^{-i \widetilde{k}^{+} \widehat{w}^{-}}\left(1+A^{n \bar{n} \cdots \bar{n}}(m-1)\left(\widetilde{k}^{+}\right)^{m-1}\right) F\left(w\left(\widehat{w}^{-} n\right)\right) .
\end{aligned}
$$

To achieve the second line above, we integrate by parts in $\widetilde{k}^{+}$, replace one power of $\widetilde{k}^{+}$ with $i \partial\left(e^{-i \widetilde{k}^{+} \widehat{w}^{-}}\right) / \partial \widehat{w}^{-}$in the term proportional to $\widehat{w}^{-}$, integrate by parts in $\widehat{w}^{-}$. These expressions demonstrate that the PDF can still be written as a regular function and that for $m=1$ it reproduces the known result for the coefficient $c_{f}^{\mu \nu}$.

To conclude the argument, we use eq. (3.28) to calculate the $n$th moment of the PDF,

$$
\begin{aligned}
\int d \widetilde{k}^{+}\left(\widetilde{k}^{+}\right)^{n} f_{f}\left(\widetilde{k}^{+}\right) & =\int d \widehat{w}^{-} \frac{d \widetilde{k}^{+}}{2 \pi} F\left(\widehat{w}^{-} n\right) e^{-i \widetilde{k}^{+} \widehat{w}^{-}}\left(\left(\widetilde{k}^{+}\right)^{n}-A^{n \bar{n} \cdots \bar{n}} n\left(\widetilde{k}^{+}\right)^{m+n-1}\right) \\
& =\int d \widehat{w}^{-} F\left(\widehat{w}^{-} n\right)\left[(+i)^{n} \delta^{(n)}\left(\widehat{w}^{-}\right)-A^{n \bar{n} \cdots \bar{n}} n(+i)^{m+n-1} \delta^{(m+n-1)}\left(\widehat{w}^{-}\right)\right] \\
& =\left.(-i)^{n} \frac{\partial^{n}}{\partial\left(\widehat{w}^{-}\right)^{n}}\left(1-n A^{n \bar{n} \cdots \bar{n}}(-i)^{m-1} \frac{\partial^{m-1}}{\partial\left(\widehat{w}^{-}\right)^{m-1}}\right) F\left(\widehat{w}^{-} n\right)\right|_{\widehat{w}^{-}=0} \\
& =\left.\left(-i \frac{\partial}{\partial \widehat{w}^{-}}-A^{n \bar{n} \cdots \bar{n}}(-i) \frac{\partial}{\partial \widehat{w}^{-}} \cdots(-i) \frac{\partial}{\partial \widehat{w}^{-}}\right)^{n} F\left(\widehat{w}^{-} n\right)\right|_{\widehat{w}^{-}=0} \\
& =\left.\left[n^{\mu}\left(-i \frac{\partial}{\partial \widehat{w}^{\mu}}-A_{\mu}^{\mu_{1} \cdots \mu_{m}}(-i) \frac{\partial}{\partial \widehat{w}^{\mu_{1}}} \cdots(-i) \frac{\partial}{\partial \widehat{w}^{\mu_{m}}}\right)\right]^{n} F\left(\widehat{w}^{-} n\right)\right|_{\widehat{w}^{-}=0} \\
& =\left.(-1)^{n}\left(n^{\mu} i \widetilde{\partial}_{\mu}\right)^{n} F\left(\widehat{w}^{-} n\right)\right|_{\widehat{w}^{-}=0} \\
& =\left.\frac{(-1)^{n}}{2} n_{\mu} n_{\mu_{1}} \cdots n_{\mu_{n}}\left\langle p\left|i \widetilde{\partial}^{\mu_{1}} \cdots i \widetilde{\partial}^{\mu_{n}} \bar{\psi}_{f}\left(\widehat{w}^{-} n\right) \gamma^{\mu} \psi_{f}(0)\right| p\right\rangle\right|_{\widehat{w}^{-}=0} \\
& =\frac{1}{2} n_{\mu} n_{\mu_{1}} \cdots n_{\mu_{n}}\left\langle p\left|\mathcal{O}_{f}^{\mu \mu_{1} \cdots \mu_{n}}\right| p\right\rangle \\
& =(n \cdot p)^{n+1} \mathcal{A}_{n+1}^{f},
\end{aligned}
$$

which is the desired result. Note that for this derivation we have implicitly worked with the spin-independent basis of operators given in eq. (3.20) to make connection with the spin-independent PDF in eq. (3.15). We anticipate that a generalization of this result holds 
for the spin-dependent PDFs for a suitable choice of operator basis. Note also that the above matching of the factorization result to the OPE means that the PDFs cannot depend on additional scalar quantities, which thereby provides support for our approach.

\subsection{Minimal $c$-type coefficients}

As a first application of the above methods, we revisit the dominant effects of minimal CPT-even Lorentz violation on the $u$ - and $d$-quark sectors in unpolarized electron-proton scattering mediated by photon exchange. In the massless limit, the relevant electromagnetic Lagrange density is [30]

$$
\mathcal{L}=\sum_{f=u, d} \frac{1}{2} \bar{\psi}_{f}\left(\eta^{\mu \nu}+c_{f}^{\mu \nu}\right) \gamma_{\mu} i \stackrel{\leftrightarrow}{D}_{\nu} \psi_{f}
$$

where $\stackrel{\leftrightarrow}{D}_{\nu}=\stackrel{\leftrightarrow}{\partial}_{\nu}+2 i e_{f} A_{\nu}$ with $e_{f}$ the quark charges. As noted in section 2 , the coefficients $c_{f}^{\mu \nu}$ are assumed symmetric and traceless.

The inclusion of dimension-four Lorentz-violating operators produces a nonhermitian hamiltonian and corresponding unconventional time evolution of the external fields [80]. One method to handle this is to perform a fermion-field redefinition to obtain a hermitian hamiltonian and hence a unitary time evolution. This induces a noncovariant relationship between spinors in different observer frames [63, 80, 81]. An alternative approach is to introduce an unconventional scalar product in Hilbert space while preserving spinor observer covariance $[67,82]$. The two approaches are known to yield equivalent physical results at leading order in Lorentz violation. We adopt the second one in this work, as it preserves the compatibility of the PDF definitions with the various observer Lorentz transformations used in the methodology developed here. Details of this quantization procedure are given in ref. [82].

The dispersion relation for eq. (3.30) is

$$
\widetilde{k}_{f}^{2}=0,
$$

where $\widetilde{k}_{f}^{\mu} \equiv\left(\eta^{\mu \nu}+c_{f}^{\mu \nu}\right) k_{\nu}$. For these coefficients, the tilde operation is linear and thus can be applied to an arbitrary set of 4 -vectors. As described in section 2, the on-shell condition (3.31) is satisfied by the parametrization $\widetilde{k}=\xi p$, where $p$ is the proton momentum. Note that this choice renders $\widetilde{k}$ independent of flavor. The physical momentum $k$ is thus given by

$$
k_{f}^{\mu}=\xi\left(p^{\mu}-c_{f}^{\mu p}\right),
$$

where $c_{f}^{\mu p} \equiv c_{f}^{\mu \nu} p_{\nu}$. Note that $k$ can differ from $\widetilde{k}$ only by possible 4 -vectors constructed from $\xi, p^{\mu}$, and $c_{f}^{\mu p}$, and the requirement (3.31) implies that the only available 4-vector in this case is $c_{f}^{\mu p}$.

The modified Breit frame fixed by $\vec{p}+\overrightarrow{\widetilde{q}}_{f}=0$ with $\widetilde{q}_{f}^{\mu}=\left(\eta^{\mu \nu}+c_{f}^{\mu \nu}\right) q_{\nu}$ is flavor dependent. However, no interference between the different flavor channels occurs at leading order because the DIS process is within the regime of incoherent scattering. Transforming to the modified Breit frame, we can apply eqs. (3.11)-(3.13) with the appropriate tilde 
operation. The scattered parton has $k^{\prime \mu}=k^{\mu}+q^{\mu}$ by construction and also satisfies $\left(\widetilde{k_{f}+q_{f}}\right)^{2}=0$, where by linearity of the tilde operation we have $\widetilde{k+q_{f}}=\widetilde{k}^{\mu}+\widetilde{q}_{f}^{\mu}$. In particular, this implies $\widetilde{q}_{f}^{\mu}=q^{\mu}+c_{f}^{\mu q}$. Note that the flavor dependence of $\widetilde{q}_{f}$ is thereby transferred to $\widetilde{k}_{f}^{\prime}$.

The unpolarized differential cross section (3.2) can be written in the form [30]

$$
\frac{d \sigma}{d x d y d \phi}=\frac{\alpha^{2} y}{2 \pi q^{4}} L_{\mu \nu} \operatorname{Im} T^{\mu \nu}
$$

where the electron tensor in the massless limit is $L^{\mu \nu}=2\left(l^{\mu} l^{\prime \nu}+l^{\nu} l^{\prime \mu}-\left(l \cdot l^{\prime}\right) \eta^{\mu \nu}\right)$, and the incident and scattered electron momenta are parametrized as $l^{\mu}=E(1,0,0,-1)$ with $l^{\prime \mu}=E^{\prime}(\sin \theta \cos \phi, \sin \theta \sin \phi, \cos \theta)$ in terms of the polar angle $\theta$ and the azimuthal angle $\phi$ defined relative to a chosen $z$ axis. The current (3.4) is a modified vector current

$$
j_{f}^{\mu}=e_{f} \bar{\psi}_{f} \Gamma_{f}^{\mu} \psi_{f},
$$

where $\Gamma_{f}^{\mu}=\left(\eta^{\mu \nu}+c_{f}^{\nu \mu}\right) \gamma_{\nu}$ with $c_{f}^{\nu \mu}$ nonzero for $f=u, d$. Since only the vector part of the interaction survives, all relevant quantities are in place to construct the explicit cross section. The forward amplitude for a single flavor $f$ reads

$$
T_{f}^{\mu \nu}=\int \frac{d \xi}{\xi} e_{f}^{2} \operatorname{Tr}\left[\Gamma_{f}^{\mu} \frac{-1}{\xi \not p+\widetilde{q} f+i \epsilon} \Gamma_{f}^{\nu} \frac{\xi \not p}{2}\right] f_{f}\left(\xi, c_{f}^{p p}\right) .
$$

Using the basis (3.16), we find $f_{f}\left(\xi, c_{f}^{p p}\right)$ is given in covariant form by

$$
f_{f}\left(\xi, c_{f}^{p p}\right)=\int \frac{d \lambda}{2 \pi} e^{-i \xi p \cdot n \lambda}\left\langle p\left|\bar{\psi}\left(\lambda \widetilde{n}_{f}\right) \frac{\not h}{2} \psi(0)\right| p\right\rangle .
$$

Note the similarity between the PDF derived in the presence of Lorentz violation and the conventional PDF in eq. (3.18). The PDF (3.36) remains independent of spin since the coefficients $c_{f}^{\mu \nu}$ control spin-independent operators in the theory. In principle, the jacobian factors $J_{k} J_{w}$ resulting from the change of integration variables contain contributions proportional to the trace of the coefficients $c_{f}^{\mu \nu}$, but the latter vanish by assumption and hence are irrelevant. The explicit dependence of the matrix elements on the coefficients for Lorentz violation arises through the shifted variable $\widetilde{n}_{f}$, which induces an implicit dependence on a single scalar quantity $c_{f}^{p p}$. Further insight on this is provided in section 3.3.

The imaginary part of the propagator denominator may be calculated using eq. (3.10), which yields

$$
2 \operatorname{Im} \frac{-1}{\left(\xi p+\widetilde{q}_{f}\right)^{2}+i \epsilon}=2 \pi \frac{\widetilde{x}_{f}}{\widetilde{Q}_{f}^{2}} \delta\left(\xi-\widetilde{x}_{f}\right),
$$

where $\widetilde{Q}_{f}^{2} \equiv-\widetilde{q}_{f}^{2}$. In this particular case, the variable $\widetilde{x}_{f}$ corresponds to the generic definition (3.12) because $\left(\xi p+\widetilde{q}_{f}\right)^{2}$ is linear in $\xi$. Using eqs. (3.35)-(3.37), we can verify the Ward identity $q_{\mu} W^{\mu \nu}=0$. This must hold here because $2 \operatorname{Im} T^{\mu \nu}=W^{\mu \nu}$ in the physical scattering region defined by $\widetilde{k}_{f}^{2}=\left(\widetilde{k}_{f}+\widetilde{q}_{f}\right)^{2}=0$ with $q^{2}<0$. Note that the Ward identity 
requires both the incident and scattered quark to be on shell. To leading order in the coefficients $c_{f}^{\mu \nu}$, we find

$$
\widetilde{x}_{f}=x\left(1+\frac{2 c_{f}^{q q}}{q^{2}}\right)+\frac{x^{2}}{q^{2}}\left(c_{f}^{p q}+c_{f}^{q p}\right) .
$$

The difference between $\widetilde{x}_{f}$ and the quantity $x_{f}^{\prime}$ in eq. (13) of ref. [30] is a single term proportional to $\xi^{2} c_{f}^{p p}$, which is removed in the current approach by the on-shell relation for the partons.

Summing over all flavors, denoting $\operatorname{Im} T^{\mu \nu}=\sum_{f} \operatorname{Im} T_{f}^{\mu \nu}$, combining eq. (3.37) with the numerator trace in eq. (3.35), integrating over $\xi$, and contracting with $L_{\mu \nu}$ gives the explicit form of the cross section as

$$
\frac{d \sigma}{d x d y d \phi}=\frac{\alpha^{2} y}{2 Q^{4}} \sum_{f} e_{f}^{2} \frac{1}{\widetilde{Q}_{f}^{2}} L_{\mu \nu} H_{f}^{\mu \nu} f_{f}\left(\widetilde{x}_{f}, c_{f}^{p p}\right),
$$

where

$$
\begin{aligned}
H_{f}^{\mu \nu} \equiv \operatorname{Tr}\left[\Gamma_{f}^{\mu}\left({\widetilde{k_{f}}}_{f}+\widetilde{q}_{f}\right) \Gamma_{f}^{\nu} \frac{\widetilde{k_{f}}}{2}\right], \\
L_{\mu \nu} H_{f}^{\mu \nu}=8\left[2\left(\widehat{k}_{f} \cdot l\right)\left(\widehat{k}_{f} \cdot l^{\prime}\right)+\widehat{k}_{f} \cdot\left(l-l^{\prime}\right)\left(l \cdot l^{\prime}\right)+2\left(\widehat{k}_{f} \cdot l\right)\left(c_{f}^{\widehat{k}_{f} l^{\prime}}+c_{f}^{l^{\prime} \widehat{k}_{f}}-c_{f}^{l^{\prime} l^{\prime}}\right)\right. \\
\left.+2\left(\widehat{k}_{f} \cdot l^{\prime}\right)\left(c_{f}^{\widehat{k}_{f} l}+c_{f}^{l \widehat{k}_{f}}+c_{f}^{l l}\right)-2\left(l \cdot l^{\prime}\right) c_{f}^{\widehat{k}_{f} \widehat{k}_{f}}\right]
\end{aligned}
$$

with $\widehat{k}_{f}^{\mu} \equiv \widetilde{x}_{f}\left(p^{\mu}-c_{f}^{\mu p}\right)$ and $\widetilde{\widehat{k}_{f}^{\mu}}=\widetilde{x}_{f} p^{\mu}$. At leading order in Lorentz violation, corrections to $\widehat{k}_{f}^{\mu}$ contribute only to the first line of eq. (3.40). Note that the sum in eq. (3.39) includes contributions from both quarks and antiquarks. Since the $c$-type coefficients control CPTeven effects, the quark and antiquark contributions are identical modulo the PDFs.

The above derivation provides an explicit demonstration that the hadronic tensor in the presence of Lorentz violation factorizes into a hard part proportional to $H_{f}^{\mu \nu}$ in eq. (3.40). This contribution to the cross section (3.39) resembles the vertex structure of an elastic partonic subprocess, which has a number of interesting implications. The covariant parametrization $\widetilde{k}^{\mu}=\xi p^{\mu}$ is so far motivated by SME considerations, factorization arguments, and the OPE approach. The cross section (3.39) is an observer scalar because it is composed of scalar kinematical objects, including the PDFs and the contraction of covariant tensor structures. Next, we demonstrate that the cross section may be decomposed into purely observer scalar quantities that can be interpreted in terms of partonic and hadronic quantities only when the choice $\widetilde{k}^{\mu}=\xi p^{\mu}$ is made. This supports the notion that, in the restricted kinematical regime of interest, the hard process can be viewed as if it were mediated by a massless on-shell SME parton scattering from the virtual photon.

To see this, consider the forward spin-averaged elastic-scattering matrix element $M$ of a virtual photon of momentum $q$ scattering from a free massless SME quark of momentum 
$k$ with flavor $f$. Using the sum over fermion spins, we find this is given by [78, 82]

$$
M=e_{f}^{2} \delta\left(\xi-\widetilde{x}_{f}\right) \frac{2 \pi \widetilde{x}_{f}}{\widetilde{Q}_{f}^{2}} \operatorname{Tr}\left[\Gamma_{f}^{\mu}\left(\widetilde{\not}_{f}+\widetilde{q}_{f}\right) \Gamma_{f}^{\nu} \frac{\widetilde{k}_{f}}{2} \frac{N(\vec{k})}{2 \widetilde{k}_{f}^{0}}\right],
$$

where $N(\vec{k})$ is the fermion-field normalization and $\widetilde{\widetilde{k}}_{f}^{\mu} \equiv k^{\mu}+2 c_{f}^{\mu k}$. Note that this result is consistent with eq. (3.35). In constructing a differential cross section that preserves Lorentz observer invariance, one typically forms the product of the differential decay rate and the initial-state flux factor. For general colliding species $A, B$, the flux factor may be expressed in terms of the beam densities $N(\vec{A}), N(\vec{B})$ and velocities $v_{A}^{j}, v_{B}^{j}$ as

$$
F=N(\vec{A}) N(\vec{B}) \sqrt{\left(\vec{v}_{A}-\vec{v}_{B}\right)^{2}-\left(\vec{v}_{A} \times \vec{v}_{B}\right)^{2}},
$$

where the group velocity $v_{A, B}^{j}$ is defined as

$$
v_{A, B}^{j}=\frac{\partial k_{A, B}^{0}}{\partial k_{A, B}^{j}} .
$$

For the $c$-type coefficients with $\widetilde{k}_{f}^{2}=0$, the group velocity is found to be [78]

$$
v_{g}^{j}=\frac{\widetilde{\tilde{k}}_{f}^{j}}{\widetilde{\widetilde{k}}_{f}^{0}}
$$

Using eqs. (3.42)-(3.43), the flux for the collision of an electron of momentum $l^{\mu}$ and a quark of momentum $k_{f}^{\mu}$ can be expressed as

$$
F=N(\vec{k}) N(\vec{l}) \frac{\sqrt{\left(\widetilde{\kappa}_{f} \cdot l\right)^{2}-\widetilde{k}_{f}^{2} l^{2}}}{\widetilde{k}_{f}^{0} l^{0}} .
$$

Combining eqs. (3.41) and (3.45) and the associated leptonic vertex contribution with spin averaging, one sees the factor $\widetilde{\widetilde{k}}_{f}^{0} l^{0}$ cancels leaving a scalar quantity. A cross section for the partonic subprocess has thus been found that constitutes a substructure of the full hadronic cross section given in eq. (3.39). The hadronic cross section may thus be expressed in terms of an integral over these partonic cross sections scaled by the ratio of the partonic flux factor to that of the convential hadronic flux factor $2 s$. This ratio is equal to unity for dimension-three operators but typically differs from unity for dimension-four and higher operators. However, it can at most produce a shift at first order in the coefficients for Lorentz violation.

In contrast, if one instead chooses the parametrization $k=\xi p$, the above construction and interpretation of the hard-scattering process cannot be made. This alternative would represent an off-shell subprocess and would spoil electromagnetic gauge invariance. It also implies that the group velocity of the parton is exactly equal to that of the hadron as usual, which prevents the cancellation of the factor of $\widetilde{\widetilde{k}}^{0}$ appearing in the trace without a concomitant unconventional redefinition of the flux. It follows that satisfactory partonic cross 
sections cannot be constructed in this alternative scenario, so a consistent interpretation of the hard scattering becomes unclear. Note that this discussion pertains only to dimensionless $c$ - and $d$-type coefficients for Lorentz violation, which produce nonscalar quantities from fermion spin sums as a consequence of the quantization procedure.

Finally, we remark that the connection between the moments of the PDFs and the matrix elements of the operators in the OPE is comparatively straightforward for the $c$ type coefficients. The $n$th moment of the PDF is

$$
\begin{aligned}
\int d \widetilde{k}^{+}\left(\widetilde{k}^{+}\right)^{n} f_{f}\left(\widetilde{k}^{+}\right) & =\int d \widehat{w}^{-}\left\langle p\left|\bar{\psi}_{f}\left(w\left(\widehat{w}^{-} n\right)\right) \frac{\not h}{2} \psi_{f}(0)\right| p\right\rangle \int \frac{d \widetilde{k}^{+}}{2 \pi}\left(\widetilde{k}^{+}\right)^{n} e^{-i \widetilde{k}^{+} \widehat{w}^{-}} \\
& =\int d \widehat{w}^{-}\left\langle p\left|\bar{\psi}_{f}(0) \frac{\not h}{2} \psi_{f}\left(-w\left(\widehat{w}^{-} n\right)\right)\right| p\right\rangle(+i)^{n} \delta^{(n)}\left(\widehat{w}^{-}\right) \\
& =\left.\left(+i^{n}\right)\left\langle p\left|\bar{\psi}_{f}(0) \frac{\not h}{2} \frac{\partial^{n}}{\partial\left(\widehat{w}^{-}\right)^{n}} \psi_{f}\left(w\left(\widehat{w}^{-} n\right)\right)\right| p\right\rangle\right|_{\widehat{w}^{-}=0} .
\end{aligned}
$$

In this case, we have

$$
\begin{aligned}
w^{\mu}\left(\widehat{w}^{-} n\right) & =\left(\eta^{\mu \nu}+c_{f}^{\mu \nu}\right) n_{\nu} \widehat{w}^{-} \\
\frac{\partial}{\partial \widehat{w}^{-}} & =\frac{\partial w^{\mu}}{\partial \widehat{w}^{-}} \frac{\partial}{\partial w^{\mu}}=n^{\mu}\left(\eta_{\mu \nu}+c_{f_{\mu \nu}}\right) \partial^{\nu}=n_{\mu} \widetilde{\partial}^{\mu},
\end{aligned}
$$

and we therefore obtain

$$
\int d \widetilde{k}^{+}\left(\widetilde{k}^{+}\right)^{n} f_{f}\left(\widetilde{k}^{+}\right)=\left.\frac{1}{2} n_{\mu} n_{\mu_{1}} \cdots n_{\mu_{n}}\left\langle p\left|\bar{\psi}_{f}(0) \gamma^{\mu} i \widetilde{\partial}^{\mu_{1}} \cdots i \widetilde{\partial}^{\mu_{n}} \psi_{f}\left(w\left(\widehat{w}^{-} n\right)\right)\right| p\right\rangle\right|_{\widehat{w}^{-}=0} .
$$

Taking advantage of the totally symmetric nature of the tensor $n_{\mu} n_{\mu_{1}} \cdots n_{\mu_{n}}$, the absence of trace contributions to the matrix element, and the replacement of regular derivatives with covariant ones as required by gauge invariance yields

$$
\int d \widetilde{k}^{+}\left(\widetilde{k}^{+}\right)^{n} f_{f}\left(\widetilde{k}^{+}\right)=\frac{1}{2} n_{\mu} \cdots n_{\mu_{n}}\left\langle p\left|\mathcal{O}_{f}^{\mu \cdots \mu_{n}}\right| p\right\rangle=(n \cdot p)^{n+1} \mathcal{A}_{n+1}^{f} .
$$

Using the moments to reconstruct the whole PDF, we see that the only dependence on the coefficients $c_{f}^{\mu \nu}$ arises from the matrix elements $\mathcal{A}_{n+1}^{f}$ and $f_{f}\left(\xi, c_{f}^{p p}\right)$. Note that the PDF is a dimensionless quantity and so $c_{f}^{p p}$ has to appear in the combination $c_{f}^{p p} / \Lambda_{\mathrm{QCD}}^{2}$, which emphasizes the genuinely nonperturbative origin of this dependence.

\subsection{Nonminimal $a^{(5)}$-type coefficients}

In the context of unpolarized DIS, the effects of nonzero flavor-diagonal quark coefficients $a_{f}^{(5) \mu \alpha \beta}$ controlling CPT-odd operators with mass dimension five have recently been studied [32]. These coefficients stem from the nonminimal SME term

$$
\mathcal{L}_{\mathrm{SME}} \supset-\left(a^{(5)}\right)_{A B}^{\mu \alpha \beta} \bar{\psi}_{A} \gamma_{\mu} i D_{(\alpha} i D_{\beta)} \psi_{B}+\text { h.c. }
$$

Nonzero proton coefficients $a_{p}^{(5) \mu \alpha \beta}$ were included in the DIS analysis of ref. [32] because current experiments constrain them only partially [11]. To avoid complications with modified 
kinematics for the external states and with the interpretation of proton matrix elements, we assume here conventional proton states so that $a_{p}^{(5) \mu \alpha \beta}=0$. Incorporating effects of nonzero proton coefficients into the following analysis is an interesting open issue but lies outside our present scope. Note that the connection between quark and proton coefficients is under investigation in the context of chiral perturbation theory [25-29] and may provide insights along these lines.

Following the method developed in section 2, the quark momentum is parametrized at leading order in Lorentz violation as

$$
k_{f}^{\mu}=\xi p^{\mu} \pm \xi^{2} a_{f}^{(5) \mu p p},
$$

where the + and - signs correspond to particles and antiparticles, respectively. This expression matches eq. (56) of ref. [32] for $a_{p}^{(5) \mu \alpha \beta}=0$. The corresponding global U(1) conserved current $j^{\mu}$ takes the form

$$
j_{f}^{\mu}=\bar{\psi}_{f}\left(\gamma^{\mu}-i a_{f}^{(5) \alpha \beta \mu} \gamma_{\alpha} \stackrel{\leftrightarrow}{\partial}_{\beta}\right) \psi_{f}
$$

where we now define

$$
\Gamma_{f}^{\mu}=\gamma^{\mu}-i a_{f}^{(5) \alpha \beta \mu} \gamma_{\alpha} \stackrel{\leftrightarrow}{\partial}_{\beta}
$$

Since the $a^{(5)}$-type coefficients control spin-independent operators and the current (3.52) is a modified vector current, only the leading-twist unpolarized $\operatorname{PDF} f_{f}(\xi)$ appears in $T^{\mu \nu}$, paralleling the case of the $c$-type coefficients. The choice (3.51) is also required to satisify the Ward identity.

Using eqs. (3.52)-(3.53) in the third term of eq. (3.9) and transforming to the modified Breit frame using eq. (3.51) for the quark momentum leads to the factorization of $T_{\mu \nu}$. After some calculation, we find the cross section to be

$$
\begin{aligned}
\frac{d \sigma}{d x d y d \phi}=\frac{\alpha^{2}}{q^{4}} \sum_{f} F_{2 f} & {\left[\frac{y s^{2}}{\pi}\left[1+(1-y)^{2}\right] \delta_{\mathrm{S} f}+\frac{y(y-2) s}{x} x_{\mathrm{S} f}\right.} \\
& -\frac{4}{x}\left(4 x^{2} a_{\mathrm{S} f}^{(5) p p l}+6 x a_{\mathrm{S} f}^{(5) l p q}+2 a_{\mathrm{S} f}^{(5) l q q}\right) \\
& +2 y\left(4 x^{2} a_{\mathrm{S} f}^{(5) p p p}+4 x a_{\mathrm{S} f}^{(5) p p q}+4 x a_{\mathrm{S} f}^{(5) l p p}+2 a_{\mathrm{S} f}^{(5) l p q}+a_{\mathrm{S} f}^{(5) p q q}\right) \\
+ & \left.\frac{4 y}{x}\left(2 x a_{\mathrm{S} f}^{(5) l l p}+a_{\mathrm{S} f}^{(5) l l q}\right)\right]
\end{aligned}
$$

where $F_{2 f}=e_{f}^{2} f_{f}\left(x_{\mathrm{S} f}^{\prime}\right) x_{\mathrm{S} f}^{\prime}$ with $x_{\mathrm{S} f}^{\prime}=x-x_{\mathrm{S} f}$ and

$$
\begin{aligned}
& \delta_{\mathrm{S} f}=\frac{\pi}{y s}\left[1+\frac{2}{y s}\left(4 x a_{\mathrm{S} f}^{(5) p p q}+2 a_{\mathrm{S} f}^{(5) p q q}+a_{\mathrm{S} f}^{(5) p q q}\right)\right], \\
& x_{\mathrm{S} f}=-\frac{2}{y s}\left(2 x^{2} a_{\mathrm{S} f}^{(5) p p q}+3 x a_{\mathrm{S} f}^{(5) p q q}+a_{\mathrm{S} f}^{(5) q q q}\right) .
\end{aligned}
$$

Note that this expression is consistent with the result obtained in ref. [32] in the limit $a_{p}^{(5) \mu \alpha \beta}=0$ once the observability of the $a^{(5)}$-type quark coefficients is taken into account. 
Note also that the shifted Bjorken variable (3.55) is distinct from the quantity $\widetilde{x}$ generically defined in eq. (3.12), which serves as a placeholder parametrization mimicking the conventional case. This contrasts with the case of $c$-type coefficients evidenced in eq. (3.37) because the imaginary part of the propagator denominator is quadratic in $\xi$. However, as described in section 3.1, the replacement $\xi \rightarrow x$ is satisfactory for terms proportional to the coefficients for Lorentz violation and yields the explicit expression for $\widetilde{q}_{f}$ defining the modified Breit frame as

$$
\widetilde{q}_{f}^{\mu}=q^{\mu}-a_{\mathrm{S} f}^{(5) \mu q q}-x\left(a_{\mathrm{S} f}^{(5) \mu p q}+a_{\mathrm{S} f}^{(5) \mu q p}\right) .
$$

It is interesting to observe that if the scattering is initiated by an antiquark as opposed to a quark, then the expression above acquires opposite signs at leading order in Lorentz violation, revealing that the modified Breit frame is both flavor and particle/antiparticle dependent. Since the $a^{(5)}$-type coefficients control CPT-odd effects, the antiquark contribution to the flavor sum can be obtained by the replacement $a_{\mathrm{S} f}^{\mu \alpha \beta} \rightarrow-a_{\mathrm{S} f}^{\mu \alpha \beta}$ for all explicit occurrences of the coefficients, convoluted with the appropriate antiquark PDFs.

For the spin-independent PDF (3.15), the explicit expression at the level of eq. (3.18) is illustrative. From general OPE considerations, the PDF can depend only on scalar combinations of $a_{f}^{(5) \mu \alpha \beta}$ and $p^{\nu}$. Furthermore, as the coefficients $a_{\mathrm{S} f}^{(5) \mu \alpha \beta}$ are symmetric and traceless, the only possible combination is $a_{\mathrm{S} f}^{(5) p p p} / \Lambda_{\mathrm{QCD}}^{2}$. Using eq. (3.28), we find

$$
f_{f}\left(\xi, a_{\mathrm{S} f}^{(5) p p p}\right)=\int \frac{d \lambda}{2 \pi} e^{-i \xi p \cdot n \lambda}\left\langle p\left|\bar{\psi}_{f}\left(\lambda n^{\mu}-a_{\mathrm{S} f}^{(5) n \mu \bar{n}} \lambda \xi p^{+}\right) \frac{\not}{2} \psi_{f}(0)\right| p\right\rangle\left(1+a_{\mathrm{S} f}^{(5) n \bar{n} \bar{n}} \xi p^{+}\right)
$$

as the explicit expression for the PDF.

\subsection{Estimated attainable sensitivities}

In this section, we obtain estimates for the sensitivities to SME coefficients that are attainable in experiments studying unpolarized electron-proton DIS. Comparable results can be expected from dedicated analyses with HERA data [12] and future EIC data [83-85]. We perform simulations with existing data and pseudodata using eq. (3.39) for the $c$-type $u$ - and $d$-quark coefficients for Lorentz violation and using eq. (3.54) for the $a^{(5)}$-type coefficients. For simplicity, the analysis neglects the intrinsic dependence of the PDFs on the SME coefficients described in sections 3.3 and 3.4 and given by eqs. (3.36) and (3.57). These effects are genuinely nonperturbative and constitute an interesting open issue for future investigation [86].

Experiments performed on the Earth at a given location are sensitive to SME coefficients as they appear in the laboratory frame. However, all laboratory frames are noninertial due to the rotation of the Earth and its revolution about the Sun. The standard frame adopted to report and compare measurements of SME coefficients for Lorentz violation [11] is the Sun-centered frame [87-89], which is approximately inertial over experimental timescales. In the Sun-centered frame, the time $T$ has origin at the vernal equinox 2000, the $Z$ axis is aligned with the Earth's rotation axis, the $X$ axis points from the Earth to the Sun at $T=0$, and the $Y$ axis completes the right-handed coordinate system. To an 
excellent approximation, the laboratory-frame coefficients are related to the coefficients in the Sun-centered frame by a rotation determined by the latitude of the experiment and by the local sidereal time $T_{\oplus}$, which is related to $T$ by an offset depending on the longitude of the laboratory [90]. Effects from the laboratory boost due to the rotation and revolution of the Earth are negligible for our present purposes. The rotation $\mathcal{R}$ from the electron-beam direction in the laboratory frame to the Sun-centered frame is given by [30, 78]

$$
\mathcal{R}=\left(\begin{array}{ccc} 
\pm 1 & 0 & 0 \\
0 & 0 & 1 \\
0 & \mp 1 & 0
\end{array}\right)\left(\begin{array}{ccc}
\cos \psi & \sin \psi & 0 \\
-\sin \psi & \cos \psi & 0 \\
0 & 0 & 1
\end{array}\right)\left(\begin{array}{ccc}
\cos \chi \cos \omega_{\oplus} T_{\oplus} & \cos \chi \sin \omega_{\oplus} T_{\oplus} & -\sin \chi \\
-\sin \omega_{\oplus} T_{\oplus} & \cos \omega_{\oplus} T_{\oplus} & 0 \\
\sin \chi \cos \omega_{\oplus} T_{\oplus} & \sin \chi \sin \omega_{\oplus} T_{\oplus} & \cos \chi
\end{array}\right) .
$$

In this expression, $\omega_{\oplus} \simeq 2 \pi /(23 \mathrm{hr} 56 \mathrm{~min})$ is the Earth's sidereal frequency. The angle $\chi$ is the colatitude of the laboratory, while $\psi$ is the orientation of the electron-beam momentum relative to the east cardinal direction. The final rotation in eq. (3.58) orients the Earthframe polar direction along the direction of the electron-beam momentum.

As a consequence of the rotation $\mathcal{R}$, most coefficients in the laboratory frame acquire sidereal-time variation at harmonics of the sidereal frequency. As described in refs. [30, 31], DIS experiments are primarily sensitive to the subset of coefficients associated with siderealtime variations because many systematic sources of uncertainty are correlated between different sidereal-time bins. We therefore focus here on estimating attainable sensitivities to this subset. For the symmetric traceless coefficients $c_{f}^{\mu \nu}$, the nine independent components in the Sun-centered frame can be chosen to have indices $T X, T Y, T Z, X X, X Y$, $X Z, Y Y, Y Z$, and $Z Z$. Of these, the components with indices $T Z, Z Z$ and the sum of components with indices $X X$ and $Y Y$ have no effect on sidereal variations because they control rotationally invariant effects in the $X-Y$ plane. We thus find that at most six independent $c$-type observables for each quark flavor can be measured using sidereal variations, so we can extract estimated sensitivities to the 12 coefficient combinations $c_{f}^{T X}, c_{f}^{T Y}, c_{f}^{X Z}$, $c_{f}^{Y Z}, c_{f}^{X Y}$, and $c_{f}^{X X}-c_{f}^{Y Y}$ with $f=u, d$. For the symmetric traceless coefficients $a_{\mathrm{S} f}^{(5) \lambda \mu \nu}$, the 16 independent components in the Sun-centered frame can be chosen to have indices TTT, TTX, TTY, TTZ, TXX, TXY, TXZ, TYY, TYZ, XXX, XXY, XXZ, XYY, $X Y Z, Y Y Y, Y Y Z$ [36]. The four combinations of components TTT, TTZ, TXX+TYY, and $Z X X+Z Y Y$ play no role in sidereal variations, leaving $12 a^{(5)}$-type observables for each quark flavor. We can therefore determine estimated sensitivities to the 24 coefficient combinations $a_{\mathrm{S} f}^{(5) T X X}-a_{S f}^{(5) T Y Y}, a_{\mathrm{S} f}^{(5) X X Z}-a_{\mathrm{S} f}^{(5) Y Y Z}, a_{\mathrm{S} f}^{(5) T X Y}, a_{\mathrm{S} f}^{(5) T X Z}, a_{\mathrm{S} f}^{(5) T Y Z}, a_{\mathrm{S} f}^{(5) X X X}$, $a_{\mathrm{S} f}^{(5) X X Y}, a_{\mathrm{S} f}^{(5) X Y Y}, a_{\mathrm{S} f}^{(5) X Y Z}, a_{\mathrm{S} f}^{(5) X Z Z}, a_{\mathrm{S} f}^{(5) Y Y Y}$, and $a_{\mathrm{S} f}^{(5) Y Z Z}$ with $f=u$, d. Inspection of these results reveals that the $c$-type coefficients control sidereal variations at frequencies up to $2 \omega_{\oplus}$, while the $a^{(5)}$-type coefficients control ones up to $3 \omega_{\oplus}$.

We discuss first the pertinent details of the HERA collider, the corresponding dataset, and the procedure to estimate sensitivities. The HERA colatitude is $\chi \simeq 34.6^{\circ}$, and the electron/positron beam orientation is $\psi \simeq 20^{\circ}$ north of east for $\mathrm{H} 1$ and $\psi \simeq 20^{\circ}$ south of west for ZEUS. This implies the minus sign in eq. (3.58) is appropriate for H1 and the plus sign for ZEUS. The data used here are combined electron- and positron-proton neutral-current measurements at an electron-beam energy of $E_{e}=27.5 \mathrm{GeV}$ and protonbeam energies of $E_{p}=920 \mathrm{GeV}, 820 \mathrm{GeV}, 575 \mathrm{GeV}$, and $460 \mathrm{GeV}$. Note that the use of 
positron-proton data is acceptable for studying both $c$ - and $a^{(5)}$-type coefficients because the associated cross sections are invariant under interchange of electrons and positrons. In total, 644 cross-section measurements at a given fixed $x$ and $Q^{2}$ value are available [12]. In extracting the estimated sensitivities, we use the procedure employed in ref. [30]. For each measurement of the cross section at a given value of $x$ and $Q^{2}$, we generate 1000 Gaussiandistributed pseudoexperiments to form a $\chi^{2}$ function, each of which describes the potential outcome of splitting the dataset into four bins in sidereal time with the requirement that the weighted average of the binned cross sections is identical to the measured one. In forming the theoretical contribution from Lorentz-violating effects to the $\chi^{2}$ distribution, we use ManeParse [91, 92] and the CT10 PDF set [93] for the quark PDFs. The desired estimated sensitivity to each coefficient is extracted independently by minimizing the $\chi^{2}$ function at the $95 \%$ confidence level and setting the other coefficients to zero in accordance with the standard procedure in the field [11]. Further details can be found in ref. [30].

For the EIC, two EIC proposals currently exist: JLEIC at JLab and eRHIC at BNL $[84,85]$. Here, we present simulations yielding estimates for sensitivities to the coefficients for Lorentz violation that can be expected after one and ten years of data taking for both JLEIC and eRHIC. The kinematical potential for each collider is expected to be different in their first stage of running. JLEIC is expected to obtain a luminosity on the order of $10^{34} \mathrm{~cm}^{-2} \mathrm{~s}^{-1}$ with an electron beam energy range of $3 \leq E_{e} \leq 12 \mathrm{GeV}$ and a proton energy range of $20 \leq E_{p} \leq 100 \mathrm{GeV}$, leading to a collider-frame energy range of roughly $15 \leq \sqrt{s} \leq 70 \mathrm{GeV}$. The JLEIC colatitude is $\chi \approx 52.9^{\circ}$ with electron-beam orientations $\psi \approx 47.6^{\circ}$ and $\psi \approx-35.0^{\circ}$ at the two collision points [83]. In contrast, during its first stage eRHIC is expected to operate at a luminosity on the order of $10^{34} \mathrm{~cm}^{-2} \mathrm{~s}^{-1}$ with a beam energy range of $5 \leq E_{e} \leq 20 \mathrm{GeV}$ and $50 \leq E_{p} \leq 250 \mathrm{GeV}$, leading to a collider-frame energy range of roughly $30 \leq \sqrt{s} \leq 140 \mathrm{GeV}$. The eRHIC colatitude is $\chi \approx 49.1^{\circ}$ and the electron-beam orientations are approximately $\psi \approx-78.5^{\circ}$ and $\psi \approx-16.8^{\circ}$ [84]. Further planned upgrades to each collider indicate a converging operational potential at the end of a ten-year time span.

To derive estimated sensitivities, datasets of simulated reduced cross sections with associated uncertainties over a range of $\left(E_{e}, E_{p}\right)$ values characteristic of the JLEIC and eRHIC are adopted. All datasets are generated using HERWIG 6.4 [94, 95] at next-toleading order, and estimates of detector systematics are based on those for the HERA collider [12]. The JLEIC dataset includes a total of 726 measurements spanning the ranges $x \in\left(9 \times 10^{-3}, 9 \times 10^{-1}\right)$ and $Q^{2} \in\left(2.5,2.2 \times 10^{3}\right) \mathrm{GeV}^{2}$, with electron-beam energies $E_{e}=5,10 \mathrm{GeV}$ and proton beam energies $E_{p}=20,60,80,100 \mathrm{GeV}$. These data have an overall point-to-point systematic uncertainty of $0.5 \%$ for $x<0.7$ and 1.5 for $x>0.7$, as well as a $1 \%$ luminosity error. The dataset for the eRHIC includes 1488 measurements spanning the ranges $x \in\left(1 \times 10^{-4}, 8.2 \times 10^{-1}\right)$ and $Q^{2} \in\left(1.3,7.9 \times 10^{3}\right) \mathrm{GeV}^{2}$, with $E_{e}=5$, $10,15,20 \mathrm{GeV}$ and $E_{p}=50,100,250 \mathrm{GeV}$. These data have an overall $1.6 \%$ point-to-point systematic uncertainty and a $1.4 \%$ luminosity error. As with the analysis of the HERA data, ManeParse and the CT10 PDF set are used for the quark PDFs. Additional details may be found in ref. [31]. 


\begin{tabular}{|c|c|c|c|c|c|}
\hline \multirow{2}{*}{$\left|c_{u}^{T X}\right|$} & \multirow{2}{*}{$\begin{array}{l}\text { HERA } \\
6.4[6.7]\end{array}$} & \multicolumn{2}{|c|}{$\begin{array}{c}\text { JLEIC eRHIC } \\
\text { one year }\end{array}$} & \multicolumn{2}{|c|}{$\begin{array}{c}\text { JLEIC } \text { eRHIC } \\
\text { ten years }\end{array}$} \\
\hline & & 1.1 [11.] & 0.26 [11.] & $0.072[9.3]$ & 0.084 [11.] \\
\hline & $6.4[6.7]$ & $1.0 \quad[10]$. & $0.19[7.7]$ & $0.062[8.5]$ & $0.058 \quad[7.9]$ \\
\hline \multirow[t]{2}{*}{$\left|c_{u}^{T Y}\right|$} & $6.4[6.7]$ & 1.1 [11.] & 0.27 [11.] & $0.069[9.4]$ & 0.085 [11.] \\
\hline & $6.4[6.7]$ & $1.0[10]$. & $0.18[7.8]$ & $0.065[8.5]$ & $0.058 \quad[7.8]$ \\
\hline \multirow[t]{2}{*}{$\left|c_{u}^{X Z}\right|$} & 32. [33.] & 1.9 [16.] & $0.36 \quad[15]$. & $0.12[16]$. & $0.11 \quad[15]$. \\
\hline & 32. [33.] & 2.2 [19.] & $0.85 \quad[35]$. & 0.14 [19.] & 0.26 [36.] \\
\hline \multirow[t]{2}{*}{$\left|c_{u}^{Y Z}\right|$} & 32. [33.] & 1.8 [16.] & $0.37 \quad[15]$. & 0.12 [16.] & $0.12 \quad[15]$. \\
\hline & 32. [33.] & 2.2 [19.] & $0.84 \quad[35]$. & 0.14 [19.] & $0.26 \quad[36]$. \\
\hline \multirow[t]{2}{*}{$\left|c_{u}^{X Y}\right|$} & 16. [16.] & $7.0 \quad[60]$. & $0.96 \quad[40]$. & $0.44 \quad[58]$. & $0.31 \quad[40]$. \\
\hline & 16. [16.] & 3.3 [28.] & $0.40 \quad[17]$. & 0.20 [27.] & 0.13 [17.] \\
\hline \multirow[t]{2}{*}{$\left|c_{u}^{X X}-c_{u}^{Y Y}\right|$} & 50. [50.] & $6.0[51]$. & 2.8 [120.] & $0.37[50]$. & 0.89 [120.] \\
\hline & 50. [50.] & 6.4 [54.] & $2.0[82]$. & $0.40 \quad[53]$. & 0.63 [82.] \\
\hline \multirow[t]{2}{*}{$\left|c_{d}^{T X}\right|$} & 26. [27.] & 4.5 [160.] & 1.1 [45.] & 0.29 [37.] & $0.34 \quad[45]$. \\
\hline & 26. [27.] & 4.0 [150.] & 0.74 [31.] & $0.25 \quad[34]$. & 0.23 [32.] \\
\hline \multirow[t]{2}{*}{$\left|c_{d}^{T Y}\right|$} & 26. [27.] & 4.3 [170.] & 1.1 [44.] & $0.27 \quad[38]$. & $0.34 \quad[45]$. \\
\hline & 26. [27.] & 4.0 [150.] & 0.73 [31.] & 0.26 [34.] & 0.23 [31.] \\
\hline \multirow[t]{2}{*}{$\left|c_{d}^{X Z}\right|$} & 130. [130.] & 7.5 [240.] & 1.4 [61.] & 0.49 [63.] & 0.45 [61.] \\
\hline & 130. [130.] & 8.7 [280.] & 3.4 [140.] & $0.54 \quad[74]$. & 1.1 [140.] \\
\hline \multirow[t]{2}{*}{$\left|c_{d}^{Y Z}\right|$} & 130. [130.] & 7.2 [240.] & $1.5[60]$. & $0.47[64]$. & 0.46 [61.] \\
\hline & 130. [130.] & 8.6 [280.] & 3.3 [140.] & 0.57 [74.] & 1.1 [140.] \\
\hline \multirow[t]{2}{*}{$\left|c_{d}^{X Y}\right|$} & 64. [64.] & 28. [880.] & 3.8 [160.] & $1.7[230]$. & 1.2 [160.] \\
\hline & 64. [64.] & 13. [410.] & $1.6[68]$. & 0.81 [110.] & $0.52 \quad$ [67.] \\
\hline \multirow[t]{2}{*}{$\left|c_{d}^{X X}-c_{d}^{Y Y}\right|$} & 200. [200.] & 24. [750.] & 11. [460.] & 1.5 [200.] & 3.5 [460.] \\
\hline & 200. [200.] & 25. [790.] & 7.8 [330.] & 1.6 [210.] & 2.5 [330.] \\
\hline
\end{tabular}

Table 1. Best attainable sensitivities from DIS to individual coefficient components $c_{u}^{\mu \nu}$ and $c_{d}^{\mu \nu}$ estimated for HERA, JLEIC, and eRHIC. All values are in units of $10^{-5}$ and reflect the orientation giving the greatest sensitivity. Results with brackets are associated with uncorrelated systematic uncertainties between binned data, while results without brackets correspond to the assumption of $100 \%$ correlation between systematic uncertainties. We provide estimated attainable sensitivities on coefficient magnitudes for both electron beam orientations, as detailed in ref. [31]. For JLEIC and eRHIC, sensitivities are listed for both one-year and ten-year data-taking configurations.

Consider first the $c$-type coefficients. A summary of the estimated attainable sensitivities is presented in table 1 , and the distribution of pseudoexperiments as a function of $x, Q^{2}, y$ for the datasets most sensititive to Lorentz violation for the particular case of the coefficient $c_{u}^{T X}$ is shown in figure 2. Overall, the HERA dataset [12] can provide sensitivity 


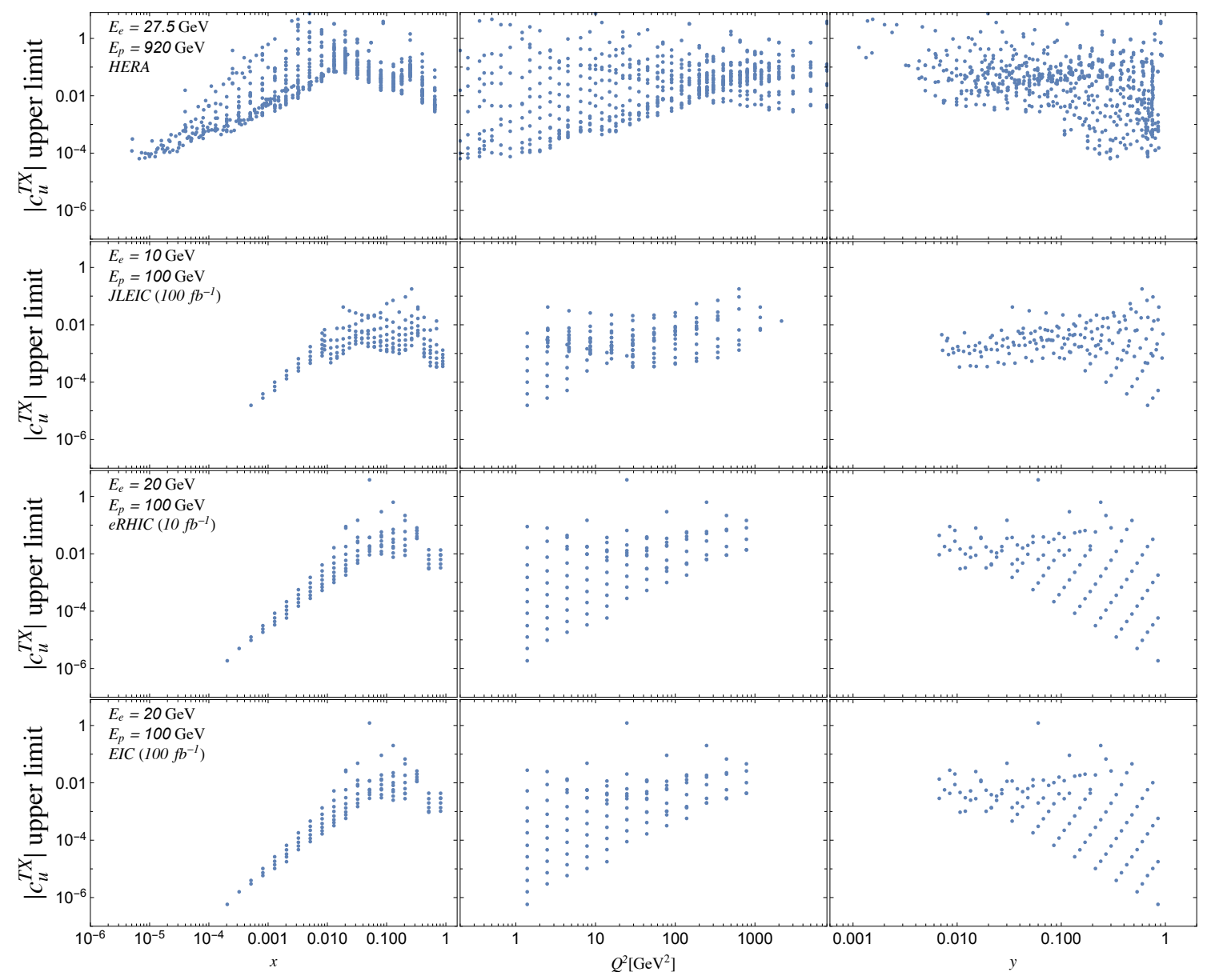

Figure 2. Distribution of $\left|c_{u}^{T X}\right|$ correlated upper sensitivities for HERA and for JLEIC and eRHIC in both initial and final configurations as functions of $x, Q^{2}$ and $y$ related through $Q^{2}=x y s$.

to Lorentz violation at roughly the $10^{-4}$ level for $u$ quarks and the $10^{-3}$ level for $d$ quarks. Both JLEIC and eRHIC can offer sensitivities at the $10^{-6}-10^{-5}$ level for $u$ quarks and the $10^{-5}-10^{-4}$ level for $d$ quarks. The reduction in senstitivity for the $d$ quark is primarily due to the difference in the squared charges $e_{u}^{2}$ and $e_{d}^{2}$. Although HERA operates at a larger collision energy and thus has a larger kinematical range, the integrated luminosity is roughly two orders of magnitude lower than that of either EIC, which leads to reduced statistics. The best attainable sensitivities appear for the low- $x$, low- $Q^{2}$, and large- $y$ region of the phase space or the deeply inelastic limit of all three colliders. The other coefficient components display a similar pattern in the distribution of sensitivities.

Intuition about the overall shape of the distribution can be gained via a plot of reduced cross sections as a function of $x$, depicted in figure 3. The increased sensitivity at low $x$ is readily apparent, with the larger CM energy for HERA implying an onset of sensitivity to Lorentz violation at lower values of $x$ than for the EIC. It is also interesting to note the similarity between the cross sections around the value of $x \sim 0.5$, which accounts for the lower sensitivity and corresponding feature seen in the distributions. The sensitivity to 


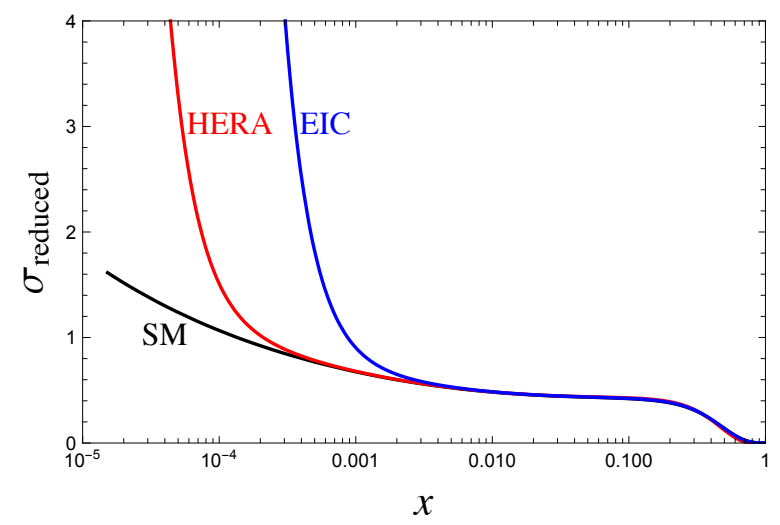

Figure 3. Comparison at fixed $Q^{2}=2 \mathrm{GeV}^{2}$ of the reduced cross sections of the leading SM contribution, of HERA at energies $E_{e}=27.5 \mathrm{GeV}, E_{p}=920 \mathrm{GeV}$, and of the EIC at energies $E_{e}=20 \mathrm{GeV}, E_{p}=100 \mathrm{GeV}$.

Lorentz violation presented here is slightly better in magnitude than the equivalent results for the EIC presented in ref. [31]. However, the distribution of sensitivities is somewhat different. In particular, the distribution is shifted to favor larger energies, with a clear preference for larger electron-beam energies and the low- $x$ region as opposed to the low- $y$ region. In addition, the grouping of the distribution is tighter and shows a clearer trend. The origin of the difference between the current and former works $[30,31]$ is due to the alteration of the on-shell condition leading to the parametrization $\widetilde{k}=\xi p$ instead of $k=\xi p$.

For the $a^{(5)}$-type coefficients, the overall estimated attainable sensitivities are presented in table 2. An illustration of a distribution of sensitivities for the coefficient component $a_{u}^{(5) T X Z}$ is displayed in figure 4. Other components have similar distributions. These results represent first estimates for the $a^{(5)}$-type quark coefficients. Overall, attainable sensitivities at the level of $10^{-6}-10^{-5} \mathrm{GeV}^{-1}$ are found for the HERA dataset and at the level of $10^{-7}-10^{-6} \mathrm{GeV}^{-1}$ for the EIC.

Notice that the overall shapes of the $c_{u}^{T X}$ distribution in figure 2 and the $a_{u}^{(5) T X Z}$ distribution in figure 4 differ in several ways. One striking feature is that the high $x$ region in the $a_{u}^{(5) T X Z}$ distribution admits the most sensitivity, particularly for the HERA data. This can be qualitatively understood as follows. As mentioned in section 3.4, the Lorentzviolating part of the cross section is proportional to the difference $f_{f}(x)-f_{\bar{f}}(x)$ between the quark and antiquark PDFs. The valence-quark content of the proton dominates the large- $x$ region, whereas the sea constituents dominate the low- $x$ region. A partial cancellation therefore occurs between the quark and antiquark contributions at very low $x$ where $f_{f}(x) \simeq f_{\bar{f}}(x)$, while the antiquark contribution has a decreased influence at large $x$. As evidenced by the JLEIC and eRHIC bounds, some sensitivity persists at low $x$. This is due to the hard contribution to the cross section (3.54), which contains terms proportional to $1 / x$. In contrast, for the $c$-type coefficients the cross section (3.39) is proportional to the sum $f_{f}(x)+f_{\bar{f}}(x)$, leading to enhanced sensitivity at low $x$. In short, the generic sensitivity to the hard contribution at low $x$, at low $Q^{2}$, and at large CM energy combine with the CPT properties to yield the predominant patterns observed in the distributions for the $c$ and $a^{(5)}$-type coefficients. 


\begin{tabular}{|c|c|c|c|c|c|}
\hline & HERA & $\begin{array}{r}\text { JLEIC } \\
\text { one }\end{array}$ & $\begin{array}{l}\text { eRHIC } \\
\text { year }\end{array}$ & $\begin{array}{c}\text { JLEIC } \\
\text { ten }\end{array}$ & $\begin{array}{l}\text { eRHIC } \\
\text { rears }\end{array}$ \\
\hline$\left|a_{\mathrm{S} u}^{(5) T X X}-a_{\mathrm{S} u}^{(5) T Y Y}\right|$ & $7.0 \quad[6.9]$ & $4.3[20]$. & 18. [20.] & 2.3 [16.] & 7.8 [20.] \\
\hline$\left|a_{\mathrm{S} u}^{(5) X X Z}-a_{\mathrm{S} u}^{(5) Y Y Z}\right|$ & 18. [18.] & $9.7[17]$. & 12. [12.] & $5.2[14]$. & 9.7 [12.] \\
\hline$\left|a_{\mathrm{Su}}^{(5) T X Y}\right|$ & $2.3[2.5]$ & $0.46[1.3]$ & $1.1[1.6]$ & $0.50[2.0]$ & $0.34[1.3]$ \\
\hline$\left|a_{\mathrm{S} u}^{(5) T X Z}\right|$ & $4.7[4.8]$ & $0.13[0.36]$ & $0.40 \quad[0.61]$ & $0.13[0.50]$ & $0.13[0.49]$ \\
\hline$\left|a_{\mathrm{Su}}^{(5) T Y Z}\right|$ & $4.6[4.8]$ & $0.12[0.37]$ & $0.40[0.61]$ & $0.13[0.50]$ & $0.13[0.48]$ \\
\hline$\left|a_{\mathrm{S} u}^{(5) X X X}\right|$ & $1.7[1.8]$ & $0.14[0.40]$ & $0.56[0.86]$ & $0.14[0.53]$ & $0.18[0.70]$ \\
\hline$\left|a_{\mathrm{Su}}^{(5) X X Y}\right|$ & $1.6[1.7]$ & $0.15[0.43]$ & $0.55[0.85]$ & $0.14[0.56]$ & $0.18[0.67]$ \\
\hline$\left|a_{\mathrm{S} u}^{(5) X Y Y}\right|$ & $1.6[1.7]$ & $0.15[0.42]$ & $0.55[0.85]$ & $0.14[0.56]$ & $0.18[0.68]$ \\
\hline$\left|a_{\mathrm{S} u}^{(5) X Y Z}\right|$ & 10. [11.] & $0.68[1.9]$ & $1.4[2.1]$ & $0.79[3.1]$ & $0.43[1.6]$ \\
\hline$\left|a_{\mathrm{S} u}^{(5) X Z Z}\right|$ & $2.1[2.2]$ & $0.12[0.34]$ & $0.39[0.60]$ & $0.12[0.45]$ & $0.13[0.48]$ \\
\hline$\left|a_{\mathrm{S} u}^{(5) Y Y Y}\right|$ & $1.7[1.7]$ & $0.14[0.41]$ & $0.56[0.87]$ & $0.14[0.53]$ & $0.18[0.68]$ \\
\hline$\left|a_{\mathrm{Su}}^{(5) Y Z Z}\right|$ & $2.1[2.1]$ & $0.12[0.35]$ & $0.39[0.60]$ & $0.12[0.46]$ & $0.12[0.47]$ \\
\hline$\left|a_{\mathrm{S} d}^{(5) T X X}-a_{\mathrm{S} d}^{(5) T Y Y}\right|$ & 110. [110.] & 70. [290.] & 360. [400.] & 24. [310.] & 83. [400.] \\
\hline$\left|a_{\mathrm{S} d}^{(5) X X Z}-a_{\mathrm{S} d}^{(5) Y Y Z}\right|$ & 300. [290.] & 160. [250.] & 240. [240.] & 56. [270.] & 180. [240.] \\
\hline$\left|a_{\mathrm{S} d}^{(5) T X Y}\right|$ & 38. [40.] & $7.4[20]$. & 21. [32.] & $9.7[38]$. & $6.6[25]$. \\
\hline$\left|a_{\mathrm{S} d}^{(5) T X Z}\right|$ & 77. [79.] & $2.0[5.5]$ & $7.7[12]$. & $2.5[9.8]$ & $2.5 \quad[9.7]$ \\
\hline$\left|a_{\mathrm{S} d}^{(5) T Y Z}\right|$ & 75. [78.] & $2.0 \quad[5.6]$ & 7.7 [12.] & $2.5 \quad[9.8]$ & $2.5 \quad[9.5]$ \\
\hline$\left|a_{\mathrm{S} d}^{(5) X X X}\right|$ & 28. [29.] & $2.3[6.2]$ & 11. [17.] & $2.7 \quad[10]$. & 3.6 [14.] \\
\hline$\left|a_{\mathrm{S} d}^{(5) X X Y}\right|$ & 26. [27.] & $2.4[6.7]$ & 11. [17.] & 2.8 [11.] & 3.4 [13.] \\
\hline$\left|a_{\mathrm{S} d}^{(5) X Y Y}\right|$ & 27. [27.] & $2.4[6.5]$ & 11. [17.] & 2.8 [11.] & 3.5 [13.] \\
\hline$\left|a_{\mathrm{S} d}^{(5) X Y Z}\right|$ & 160. [170.] & 11. [29.] & 27. [40.] & 15. [60.] & $8.4[31]$. \\
\hline$\left|a_{\mathrm{S} d}^{(5) X Z Z}\right|$ & 35. [35.] & $1.9[5.2]$ & $7.6[12]$. & $2.3[8.9]$ & $2.5 \quad[9.4]$ \\
\hline$\left|a_{\mathrm{S} d}^{(5) Y Y Y}\right|$ & 27. [28.] & $2.3 \quad[6.2]$ & 11. [17.] & $2.6 \quad[10]$. & 3.5 [13.] \\
\hline$\left|a_{\mathrm{Sd}}^{(5) Y Z Z}\right|$ & 34. [35.] & $1.9[5.3]$ & 7.5 [12.] & $2.3[8.9]$ & $2.4[9.2]$ \\
\hline
\end{tabular}

Table 2. Best attainable sensitivities from DIS to individual coefficient components $a_{\mathrm{Su}}^{(5) \lambda \mu \nu}$ and $a_{\mathrm{S} d}^{(5) \lambda \mu \nu}$ estimated for HERA, JLEIC, and eRHIC. All values are in units of $10^{-6} \mathrm{GeV}^{-1}$ and reflect the orientation giving the greatest sensitivity. Results with brackets are associated with uncorrelated systematic uncertainties between binned data, while results without brackets correspond to the assumption of $100 \%$ correlation between systematic uncertainties. We provide estimated attainable sensitivities on coefficient magnitudes for both electron beam orientations, as detailed in ref. [31]. For JLEIC and eRHIC, sensitivities are listed for both one-year and ten-year data-taking configurations. 


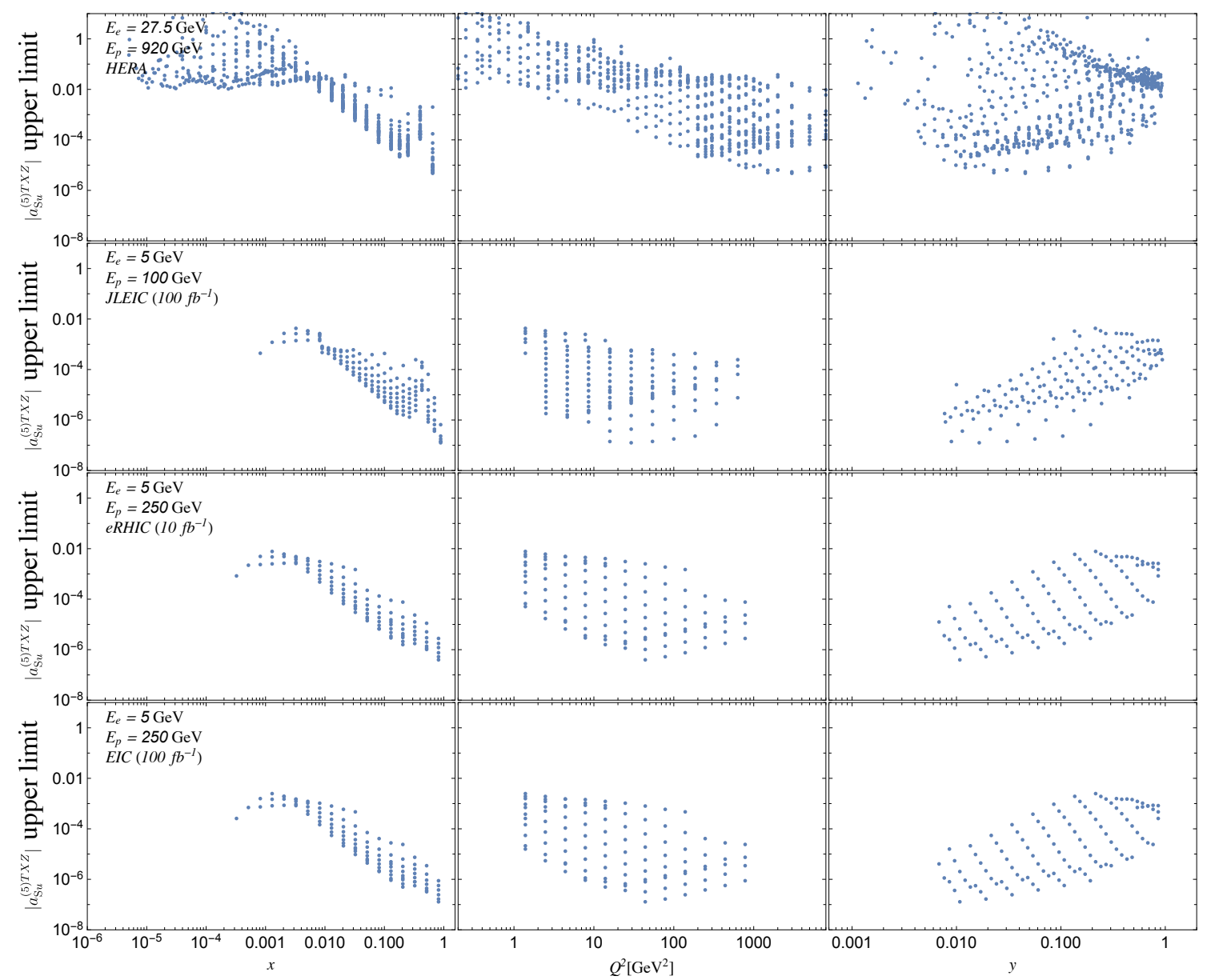

Figure 4. Distribution of $\left|a_{\mathrm{S} u}^{(5) T X Z}\right|$ correlated upper sensitivities for HERA and for JLEIC and eRHIC in both initial and final configurations as functions of $x, Q^{2}$ and $y$ related through $Q^{2}=x y s$.

\section{The Drell-Yan process}

Next, we turn attention to studying corrections from Lorentz violation to the DY process, using an analogous approach to that adopted above for DIS. The DY process involves the interaction of two hadrons leading to lepton-pair production, $H_{1}+H_{2} \rightarrow l_{1}+l_{2}+X$, where all final hadronic states $X$ are summed over and the polarizations of the final-state leptons are averaged because they are unobserved.

The total cross section is given by

$$
\sigma=\frac{1}{2 s} \int \frac{d^{3} l_{1}}{(2 \pi)^{3} 2 l_{1}{ }^{0}} \frac{d^{3} l_{2}}{(2 \pi)^{3} 2 l_{2}{ }^{0}} \sum_{X} \prod_{i=1}^{n_{X}} \frac{d^{3} p_{i}}{(2 \pi)^{3} 2 p_{i}{ }^{0}}\left|\left\langle l_{1}, l_{2}, X|\widehat{T}| p_{1}, s_{1}, p_{2}, s_{2}\right\rangle\right|^{2},
$$

where $\left|\left\langle l_{1}, l_{2}, X|\widehat{T}| p_{1}, s_{1}, p_{2}, s_{2}\right\rangle\right|^{2} \equiv(2 \pi)^{4} \delta^{4}\left(p_{1}+p_{2}-l_{1}-l_{2}-p_{X}\right)|\mathcal{M}|^{2}, p_{X} \equiv \Sigma_{i=1}^{n_{X}} p_{i}$, and $q=l_{1}+l_{2}$. We must consider all $n_{X}$ possible final hadronic states in the process because $X$ is unobserved. Note that the lepton spin labels are suppressed as they are summed over. The factor $1 / 2 s$ is the usual hadronic flux factor. 


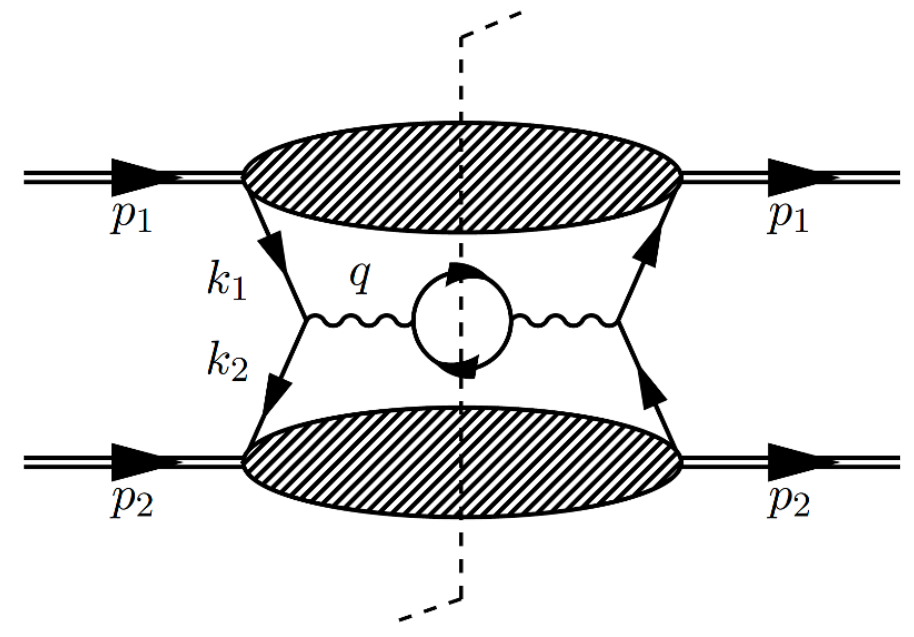

Figure 5. The dominant contribution to the DY hadronic tensor (4.3). The dashed line bisecting the graph denotes a sum over all unobserved hadronic final states.

As with DIS, our treatment considers effects of Lorentz violation on $\mathcal{M}$ and in particular on the hadronic contribution. Since this process represents the head-on collision between two hadrons, it is simplest to work in the hadron-hadron CM frame with $\vec{p}_{1}+\vec{p}_{2}=\overrightarrow{0}$. The differential cross section then takes the form

$$
d \sigma=\frac{\alpha^{2}}{2 s} \frac{1}{q^{4}} d^{4} q \frac{d \Omega_{l}}{(2 \pi)^{4}} \sum_{i} R_{i}\left(L_{i}\right)_{\mu \nu}\left(W_{i}\right)^{\mu \nu},
$$

where $i$ denotes the sum over channels with ratios $R_{i}$ to the photon propagator. The momentum $q$ is $q=l_{1}+l_{2}$, and the difference $l=l_{1}-l_{2}$ has solid angle $d \Omega_{l}$ about the lepton-pair CM. Note that $q^{2}>0$ for this process, in contrast to DIS where $q^{2}<0$.

\subsection{Factorization of the hadronic tensor}

The object of primary interest in eq. (4.2) is the hadronic tensor $W_{\mu \nu}$, which may be written as

$$
W_{\mu \nu}=\int d^{4} x e^{-i q \cdot x}\left\langle p_{1}, s_{1}, p_{2}, s_{2}\left|j_{\mu}^{\dagger}(x) j_{\nu}(0)\right| p_{1}, s_{1}, p_{2}, s_{2}\right\rangle .
$$

The dominant contribution to this object is displayed in figure 5. The current product $j_{\mu}^{\dagger}(x) j_{\nu}(0)$ can be decomposed in a similar way as done for DIS. However, we consider here the simple product of currents instead of a time-ordered product, as the latter offers no advantage for this process. We are again interested in the dominant effects of Lorentz violation at large $q^{2} \equiv Q^{2}>0$. This contains numerous Dirac structures with certain combinations dominating at the leading power in $Q$. Equation (4.3) is to be evaluated in the CM frame of the hadron-hadron collision. Given the high energy of this process in the massless hadron limit, we can parametrize without loss of generality the hadron momenta as $p_{1}=p_{1}^{+} \bar{n}$ and $p_{2}=p_{2}^{-} n$, where $\bar{n}$ and $n$ are given in eq. (3.16). Employing similar considerations as in section 3.1, this implies that the dominant Dirac structures are 
proportional to $\left\{\gamma^{-}, \gamma^{-} \gamma_{5}, \gamma^{-} \gamma_{\perp}^{i} \gamma_{5}\right\}$ and $\left\{\gamma^{+}, \gamma^{+} \gamma_{5}, \gamma^{+} \gamma_{\perp}^{i} \gamma_{5}\right\}$ for $H_{1}$ and $H_{2}$, respectively. Considering eq. (3.4), this leads to nine Dirac bilinear products constituting the leadingpower behavior of $W^{\mu \nu}$,

$$
\begin{aligned}
W^{\mu \nu} \simeq & -\frac{1}{16} \frac{1}{3} \int d^{4} x e^{-i q \cdot x} \operatorname{Tr}\left[\gamma ^ { - } \left(\left\langle p_{1}, s_{1}\left|\bar{\chi}(x) \gamma^{+} \chi(0)\right| p_{1}, s_{1}\right\rangle\right.\right. \\
& \left.+\gamma_{5}\left\langle p_{1}, s_{1}\left|\bar{\chi}(x) \gamma_{5} \gamma^{+} \chi(0)\right| p_{1}, s_{1}\right\rangle+\gamma_{5} \gamma_{\perp}^{i}\left\langle p_{1}, s_{1}\left|\bar{\chi}(x) \gamma^{+} \gamma_{\perp}^{i} \gamma_{5} \chi(0)\right| p_{1}, s_{1}\right\rangle\right) \Gamma^{\mu} \\
& \times \gamma^{+}\left(\left\langle p_{2}, s_{2}\left|\bar{\psi}(0) \gamma^{-} \psi(x)\right| p_{2}, s_{2}\right\rangle+\gamma_{5}\left\langle p_{2}, s_{2}\left|\bar{\psi}(0) \gamma_{5} \gamma^{-} \psi(x)\right| p_{2}, s_{2}\right\rangle\right. \\
& \left.\left.+\gamma_{5} \gamma_{\perp}^{i}\left\langle p_{2}, s_{2}\left|\bar{\psi}(0) \gamma^{-} \gamma_{\perp}^{i} \gamma_{5} \psi(x)\right| p_{2}, s_{2}\right\rangle\right) \Gamma^{\nu}\right]
\end{aligned}
$$

The factor of $1 / 3$ comes from the Fierz decomposition of the $\mathrm{su}(3)$ color algebra, which fixes the matrix elements into color-neutral combinations. Note also that the electroweak charges are implicit in the definitions of $\Gamma^{\mu}, \Gamma^{\nu}$.

From eq. (4.4), we define functions that are momentum-space Fourier components $k_{1}$, $k_{2}$ of the hadron matrix elements. Expressing the matrix elements in momentum space and performing the integrations over the spatial variable $x$ yields a four-dimensional delta function $\delta^{4}\left(q-k_{1}-k_{2}\right)$. Since the physical internal momenta $k_{1}, k_{2}$ are off shell in this context, a change of variables to tilde momenta must be performed. Unlike in DIS, one may work here in the conventional CM frame because the collinear component of the quark momentum comes with $\widetilde{k}$ instead of $k$. The change of variables $k_{i} \rightarrow \widetilde{k}_{i}$ produces jacobian factors $J_{k_{1}}, J_{k_{2}}$. The delta function can be expressed as

$$
\delta^{4}\left(q-k_{1}\left(\widetilde{k}_{1}\right)-k_{2}\left(\widetilde{k}_{2}\right)\right)=\delta^{4}\left(\widetilde{q}-\widetilde{k}_{1}-\widetilde{k}_{2}\right),
$$

which defines $\widetilde{q}$ for the DY process. This quantity typically depends on $k_{1}$ and $k_{2}$. Additional care is required here because one momentum obeys a modified particle dispersion relation while the other obeys the antiparticle relation. Performing a subsequent Fourier transform in the spatial variables $w_{1}, w_{2}$ followed by a change of variables to $\widehat{w}_{1}, \widehat{w}_{2}$ such that $k_{i}\left(\widetilde{k}_{i}\right) \cdot w_{i}=\widetilde{k}_{i} \cdot \widehat{w}_{i}$ as discussed in section 3.1 , we obtain the generic contribution to $W^{\mu \nu}$ in the form

$$
\begin{aligned}
\int d^{4} \widetilde{k}_{1} d^{4} \widetilde{k}_{2} d^{4} \widehat{w}_{1} d^{4} \widehat{w}_{2} J_{k_{1}} J_{k_{2}} J_{w_{1}} J_{w_{2}} H^{\text {new }}\left(\widetilde{k}_{1}, \widetilde{k}_{2}\right) \\
\times F\left(w_{1}\left(\widehat{w}_{1}\right), p_{1}\right) \bar{F}\left(w_{2}\left(\widehat{w}_{2}\right), p_{2}\right) e^{-i\left(\widetilde{k}_{1} \cdot \widehat{w}_{1}+\widetilde{k}_{2} \cdot \widehat{w}_{2}\right)}
\end{aligned}
$$

In this expression, $H^{\text {new }}\left(\widetilde{k}_{1}, \widetilde{k}_{2}\right)$ represents a Dirac structure combined with eq. (4.5). Note that this is a new function due to the change of variables on the functional form of the momentum contractions with the Dirac matrices.

Equation (4.6) resembles two copies of the analogous DIS result, cf. eqs. (3.9)-(3.9). Appealing to our interest in the leading-twist contributions and considering the portion of $W^{\mu \nu}$ constrained by $q^{\mu}$, we can approximate $H^{\text {new }}\left(\widetilde{k}_{1}, \widetilde{k}_{2}\right) \approx H^{\text {new }}\left(\widetilde{k}_{1}^{+}, \widetilde{k}_{2}^{-}\right)$, which is the leading term in the collinear expansion of the hard-scattering function [96]. In the kinematics of choice defining the magnitude and direction of $p_{1}$ and $p_{2}$, we have $\widetilde{k}_{1}^{\mu} \simeq\left(\widetilde{k}_{1}^{+}, 0, \mathbf{0}_{\perp}\right)$ and $\widetilde{k}_{2}^{\mu} \simeq\left(0, \widetilde{k}_{2}^{-}, \mathbf{0}_{\perp}\right)$ in the approximation of the hard-scattering function $H^{\text {new }}\left(\widetilde{k}_{1}^{+}, \widetilde{k}_{2}^{-}\right)$. 
The dominant portion of the term eq. (4.6) is thus

$$
\begin{aligned}
& \int d \widetilde{k}_{1}^{+} d \widetilde{k}_{2}^{-} H^{\text {new }}\left(\widetilde{k}_{1}^{+}, \widetilde{k}_{2}^{-}\right) \\
& \quad \times \int d \widetilde{k}_{1}^{-} \widetilde{k}_{1_{\perp}} d \widetilde{k}_{2}^{+} \widetilde{k}_{2_{\perp}} d^{4} \widehat{w}_{1} d^{4} \widehat{w}_{2} F\left(w_{1}\left(\widehat{w}_{1}\right), p_{1}\right) \bar{F}\left(w_{2}\left(\widehat{w}_{2}\right), p_{2}\right) e^{-i\left(\widetilde{k}_{1} \cdot \widehat{w}_{1}+\widetilde{k}_{2} \cdot \widehat{w}_{2}\right)} .
\end{aligned}
$$

The placeholder functions $F$ and $\bar{F}$ are identified with the particle and antiparticle counterparts of the PDFs derived in the case of DIS, eq. (3.15).

With the above considerations, we obtain the final form of $W^{\mu \nu}$ as

$$
\begin{aligned}
W_{f}^{\mu \nu}= & \frac{1}{4} \frac{1}{3} \frac{1}{p_{1}^{+} p_{2}^{-}} \int d \xi_{1} d \xi_{2} \delta^{4}\left(\widetilde{q}\left(q, \xi_{1} p_{1}, \xi_{2} p_{2}\right)-\xi_{1} p_{1}-\xi_{2} p_{2}\right) \\
& \times \operatorname{Tr}\left[\not p_{1}\left(\mathbb{1} f_{f}\left(\xi_{1}\right)+\gamma_{5} \lambda_{1} \Delta f_{f}\left(\xi_{1}\right)+\gamma_{5} \gamma_{\perp}^{i} \lambda_{1 \perp} \Delta_{\perp} f_{f}\left(\xi_{1}\right)\right) \Gamma^{\mu}\left(\xi_{1} p_{1}, \xi_{2} p_{2}\right)\right. \\
& \left.\quad \times \not p_{2}\left(\mathbb{1} f_{\bar{f}}\left(\xi_{2}\right)-\gamma_{5} \lambda_{2} \Delta f_{\bar{f}}\left(\xi_{2}\right)+\gamma_{5} \gamma_{\perp}^{i} \lambda_{2 \perp} \Delta_{\perp} f_{\bar{f}}\left(\xi_{2}\right)\right) \Gamma^{\nu}\left(\xi_{1} p_{1}, \xi_{2} p_{2}\right)\right],
\end{aligned}
$$

where $\widetilde{k}_{1}^{+}=\xi_{1} p_{1}^{+}$and $\widetilde{k}_{2}^{-}=\xi_{2} p_{2}^{-}$. Note the minus sign in front of the antiparton PDF, which is required for a consistent interpretation of the helicity asymmetry of the target state and the suppression of any potential implicit dependence on Lorentz violation. Also note that the matrices $\Gamma^{\mu}$ can be expressed as matrix functions of $\xi_{1} p_{1}, \xi_{2} p_{2}$ because $k, \widetilde{k}$ can be taken equal when contracted with the coefficients for Lorentz violation. Since $\widetilde{q}$ is a nonlinear function of $\widetilde{k}_{1}$ and $\widetilde{k}_{2}$, the integration over $\xi_{1}, \xi_{2}$ in eq. 4.8 is awkward. However, an integration over $d^{4} q$ is required in calculating the total cross section and so $q$ can be parametrized as usual,

$$
q^{\mu}=x_{1} p_{1}^{\mu}+x_{2} p_{2}^{\mu}+q_{\perp}^{\mu}
$$

which implies $d^{4} q=p_{1}^{+} p_{2}^{-} d x_{1} d x_{2} d q_{\perp}^{2}$. Since the argument of the delta function in eq. (4.8) is then linear in $x_{1}, x_{2}$, and $q_{\perp}$, integration can instead first be performed over the latter variables, setting $x_{i} \approx \xi_{i}$ at leading order in Lorentz violation and thus fixing $\widetilde{q}$.

Overall, we can thus conclude that the basic ideas leading to the factorization of the forward amplitude $T^{\mu \nu}$ in DIS also lead to the factorization of $W^{\mu \nu}$ for the DY process. The hadronic tensor as expressed in eq. (4.8) is in a form suitable for insertion into the differential cross section (4.2). Performing the integrations sets the momentum fractions of the partipating partons equal to the fractions of $\widetilde{q}^{ \pm}$and $\widetilde{q}_{\perp}=0$. Notice that in the above discussion we consider the situation of $k_{1}$ emanating from $p_{1}$ and $k_{2}$ from $p_{2}$. However, we must also include $k_{2}$ emanating from $p_{1}$ and $k_{1}$ from $p_{2}$. These contribute to the probability rather than to the amplitude and so represent another example of the incoherence of the partonic scattering. Note that each contribution separately satisfies the condition for electromagnetic gauge invariance.

\subsection{Minimal $c$-type coefficients}

As a first application of the above methodology, we study the implications of Lorentz violation described by eq. (3.30) on the unpolarized DY process at leading order in electromagnetic interactions. The final-state lepton pair now represents electrons, muons, or taus 
and their antiparticles. The only Dirac structure appearing is the vector current eq. (3.34), with $\Gamma_{f}^{\mu}=\left(\eta^{\mu \nu}+c_{f}^{\nu \mu}\right) \gamma_{\nu}$. In this limit, the hadronic tensor eq. (4.3) reads

$$
W_{f}^{\mu \nu}=-\frac{1}{48} e_{f}^{2} \operatorname{Tr}\left[\Gamma_{f}^{\mu} \gamma^{\rho} \Gamma_{f}^{\nu} \gamma^{\sigma}\right] \int d^{4} x e^{-i q \cdot x}\left\langle p_{1}\left|\bar{\psi}_{f}(x) \gamma_{\rho} \psi_{f}(0)\right| p_{1}\right\rangle\left\langle p_{2}\left|\bar{\psi}_{f}(0) \gamma_{\sigma} \psi_{f}(x)\right| p_{2}\right\rangle
$$

Both the interacting parton and antiparton have parametrized momenta $k_{i f}^{\mu}=\xi_{i}\left(p_{i}^{\mu}-c_{f}^{\mu p_{i}}\right)$ where $i=1,2$. By performing the factorization procedure outlined in the previous section, we obtain

$$
W_{f}^{\mu \nu}=\int d \xi_{1} d \xi_{2} H_{f}^{\mu \nu}\left(\xi_{1}, \xi_{2}\right)\left[f_{f}\left(\xi_{1}, c_{f}^{p_{1} p_{1}}\right) f_{\bar{f}}\left(\xi_{2}, c_{f}^{p_{2} p_{2}}\right)+f_{f}\left(\xi_{2}, c_{f}^{p_{2} p_{2}}\right) f_{\bar{f}}\left(\xi_{1}, c_{f}^{p_{1} p_{1}}\right)\right],
$$

where the contribution to the hard-scattering function is

$$
\begin{aligned}
& H_{f}^{\mu \nu}\left(\xi_{1}, \xi_{2}\right)=\frac{2 e_{f}^{2}}{3 \widetilde{s}} \operatorname{Tr}\left[\left(\eta^{\mu \alpha}+c_{f}^{\alpha \mu}\right) \gamma_{\alpha} \frac{\xi_{1} \not p_{1}}{2}\left(\eta^{\nu \beta}+c_{f}^{\beta \nu}\right) \gamma_{\beta} \frac{\xi_{2} \not p_{2}}{2}\right] \\
& \times(2 \pi)^{4} \delta^{4}\left(q^{\mu}+\xi_{1} c_{f}^{\mu p_{1}}+\xi_{2} c_{f}^{\mu p_{2}}-\xi_{1} p_{1}^{\mu}-\xi_{2} p_{2}^{\mu}\right),
\end{aligned}
$$

with $\widetilde{s} \equiv 2 \widetilde{k}_{1} \cdot \widetilde{k}_{2}, \widetilde{q}_{f}^{\mu}=\left(\eta^{\mu \alpha}+c_{f}^{\mu \alpha}\right) q_{\alpha}$. In adding the extra diagram, we have employed the symmetry $H_{f}^{\mu \nu}\left(\widetilde{k}_{1}^{+}, \widetilde{k}_{2}^{-}\right)=H_{f}^{\mu \nu}\left(\widetilde{k}_{2}^{-}, \widetilde{k}_{1}^{+}\right)$in eq. (4.11). The expression (4.12) is similar to the conventional result for the partonic subprocess, and the discussion of section 3.3 applies with $l \leftrightarrow \widetilde{k}_{2}$. As expected, direct calculation shows this result satisfies the electromagnetic Ward identity, $q_{\mu} W^{\mu \nu}=0$.

The unpolarized parton and antiparton PDFs are the only ones emerging in this process. The parton PDF takes the form (3.36) found for DIS. The antiparton PDF has the definition

$$
\bar{f}_{f}\left(\xi, c_{f}^{p p}\right)=-\int \frac{d \lambda}{2 \pi} e^{+i \xi p \cdot n \lambda}\left\langle p\left|\bar{\psi}(\lambda \widetilde{n}) \frac{\not h}{2} \psi(0)\right| p\right\rangle,
$$

and satisfies $\bar{f}_{f}\left(\xi, c_{f}^{p p}\right)=-f_{f}\left(-\xi, c_{f}^{p p}\right)$. Notice here that the antiparticle PDF $f_{\bar{f}}$ has the same implicit dependence on the coefficients as the particle PDF because the $c$-type coefficients affect particles and antiparticles in the same way. Contracting the leptonic and hadronic tensors then yields the total cross section as

$$
\begin{aligned}
& \sigma=\frac{2 \alpha^{2}}{3 s} \frac{1}{Q^{4}} \int d \Omega_{l} \frac{d \xi_{1}}{\xi_{1}} \frac{d \xi_{2}}{\xi_{2}} \sum_{f} e_{f}^{2}\left(\widetilde{k}_{1} \cdot l_{1}\right)\left(\widetilde{k}_{2} \cdot l_{2}\right)+\left(\widetilde{k}_{1} \cdot l_{2}\right)\left(\widetilde{k}_{2} \cdot l_{1}\right) \\
&+\left(\widetilde{k}_{1} \cdot l_{1}\right)\left(c_{f}^{\widetilde{k}_{2} l_{2}}+c_{f}^{l_{2} \widetilde{k}_{2}}\right)+\left(\widetilde{k}_{1} \cdot l_{2}\right)\left(c_{f}^{\widetilde{k}_{2} l_{1}}+c_{f}^{l_{1} \widetilde{k}_{2}}\right) \\
&+\left(\widetilde{k}_{2} \cdot l_{1}\right)\left(c_{f}^{\widetilde{k}_{1} l_{2}}+c_{f}^{l_{2} \widetilde{k}_{1}}\right)+\left(\widetilde{k}_{2} \cdot l_{2}\right)\left(c_{f}^{\widetilde{k}_{1} l_{1}}+c_{f}^{l_{1} \widetilde{k}_{1}}\right) \\
&\left.-\left(\widetilde{k}_{1} \cdot \widetilde{k}_{2}\right)\left(c_{f}^{l_{1} l_{2}}+c_{f}^{l_{2} l_{1}}\right)-\left(l_{1} \cdot l_{2}\right)\left(\widetilde{k}_{f} \widetilde{k}_{2}+c_{f}^{\widetilde{k}_{2} \widetilde{k}_{1}}\right)\right] \\
& \times\left(f_{f}\left(\xi_{1}, c_{f}^{p_{1} p_{1}}\right) f_{\bar{f}}\left(\xi_{2}, c_{f}^{p_{2} p_{2}}\right)+f_{f}\left(\xi_{2}, c_{f}^{p_{2} p_{2}}\right) f_{\bar{f}}\left(\xi_{1}, c_{f}^{p_{1} p_{1}}\right)\right) .
\end{aligned}
$$


Next, we make the kinematics explicit by parametrizing the colliding proton momenta as $p_{1}^{\mu}=E_{p}(1,0,0,1)$ and $p_{2}^{\mu}=E_{p}(1,0,0,-1)$, with $E_{p} \simeq|\vec{p}|$ and the final lepton momenta as $l_{1}^{\mu}=E_{e}(1, \sin \theta \cos \phi, \sin \theta \sin \phi, \cos \theta), l_{2}^{\mu}=E_{e}(1,-\sin \theta \cos \phi,-\sin \theta \sin \phi,-\cos \theta)$. Here, $\theta$ and $\phi$ are the usual polar and azimuthal angles with respect to the laboratory $z$ axis, chosen along the direction of motion of the two initial protons. The total CM energy is $s=\left(p_{1}+p_{2}\right)^{2}=4 E_{p}^{2}$. After performing the solid-angle integration, we find that eq. (4.14) becomes

$$
\sigma=\frac{1}{3} \int d x_{1} d x_{2} \sum_{f}\left[f_{f}\left(x_{1}\right) f_{\bar{f}}\left(x_{2}\right)+f_{f}\left(x_{2}\right) f_{\bar{f}}\left(x_{1}\right)\right] \sigma_{f}\left(\widehat{s}, c_{f}^{\mu \nu}\right) .
$$

Following the discussion in section 3.3, we have defined the equivalent partonic cross section as

$$
\sigma_{f}\left(\widehat{s}, c_{f}^{\mu \nu}\right)=\frac{4 \pi \alpha^{2} e_{f}^{2}}{3 \widehat{s}}\left(1+c_{f}^{33}-c_{f}^{00}\right)
$$

where $\widehat{s}$ is the invariant mass $s=\left(l_{1}+l_{2}\right)^{2}=\left(k_{1}+k_{2}\right)^{2}$ of the lepton pair. In this last expression for the cross section, we suppress the dependence on $c_{f}^{p_{1} p_{1}}$ and $c_{f}^{p_{2} p_{2}}$ for brevity.

The cross section as a function of $Q^{2}$ and other kinematical invariants is of interest because it is measured in experiments. In forming $d \sigma / d Q^{2}$ in a given frame, the results (4.14) and (4.15) must be converted using a delta function $\delta\left(Q^{2}-\widehat{s}\right)$. In the presence of Lorentz violation, this quantity may differ from the usual value $x_{1} x_{2} s$, so upon integration over $x_{1}$ and $x_{2}$ the PDFs may be constrained away from the normal condition $Q^{2}=x_{1} x_{2} s$ at first order in the coefficients for Lorentz violation. Note that $0 \leq x_{1}$ and $x_{2} \leq 1$, as dictated by the external kinematics. This introduces yet another way in which Lorentz-violating effects can manifest themselves in observables of interest. In the cases that follow, we find that this shift in the delta-function argument leads to the dominant source of sensitivity to Lorentz violation in the DY process.

Explicitly, we find $\widehat{s} \equiv Q^{2}=\left(k_{1}+k_{2}\right)^{2}$ has the expression

$$
\widehat{s}=x_{1} x_{2} s\left[1-\frac{1}{2 x_{1} x_{2}}\left(\left(x_{1}+x_{2}\right)^{2} c_{f}^{00}+\left(x_{1}-x_{2}\right)^{2} c_{f}^{33}-\left(x_{1}^{2}-x_{2}^{2}\right)\left(c_{f}^{03}+c_{f}^{30}\right)\right)\right],
$$

which shifts the evaluation of the derivatives. After some calculation, we find

$$
\begin{aligned}
\frac{d \sigma}{d Q^{2}}= & \frac{4 \pi \alpha^{2}}{9 Q^{4}} \sum_{f} e_{f}^{2}\left[\int_{\tau}^{1} d x \frac{\tau}{x}\left[1+2\left(1+\frac{x^{2}}{\tau}\right) c_{f}^{00}\right]\left(f_{f}(x) f_{\bar{f}}(\tau / x)+f_{f}(\tau / x) f_{\bar{f}}(x)\right)\right. \\
& \left.+\int_{\tau}^{1} d x \frac{\tau}{x}\left[\left(x-\frac{\tau}{x}\right) c_{f}^{33}+\left(x+\frac{\tau}{x}\right) c_{f}^{00}\right]\left(f_{f}(x) f_{\bar{f}}^{\prime}(\tau / x)+f_{f}^{\prime}(\tau / x) f_{\bar{f}}(x)\right)\right]
\end{aligned}
$$

where $\tau \equiv Q^{2} / s$ is the usual scaling variable with $0 \leq \tau \leq 1$. Here, the notation $f^{\prime}(y)$ denotes the derivative of the PDF evaluated at $y$.

From the expression (4.18), we see that only the single coefficient $c_{f}^{33}$ controls the sidereal-time dependence of the cross section, since $c_{f}^{00}$ is invariant under rotations. The term $c_{f}^{03}=c_{f}^{30}$ is absent because it is multiplied by a factor $\left(x_{1}^{2}-x_{2}^{2}\right)$ that is antisymmetric 
in $x_{1} \leftrightarrow x_{2}$, while the cross section is symmetric under this interchange. The result (4.17) also has the interesting feature of being independent of time whenever $x_{1}=x_{2}$. The time dependence can be explicitly revealed by expressing the single laboratory-frame coefficient controlling the time dependence in terms of coefficients in the Sun-centered frame,

$$
\begin{aligned}
c_{f}^{33} & =c_{f}^{X X}\left(\cos \chi \sin \psi \cos \Omega_{\oplus} T_{\oplus}+\cos \psi \sin \Omega_{\oplus} T_{\oplus}\right)^{2} \\
& +c_{f}^{Y Y}\left(\cos \chi \sin \psi \sin \Omega_{\oplus} T_{\oplus}-\cos \psi \cos \Omega_{\oplus} T_{\oplus}\right)^{2} \\
& +2 c_{f}^{X Y}\left(\cos \chi \sin \psi \cos \Omega_{\oplus} T_{\oplus}+\cos \psi \sin \Omega_{\oplus} T_{\oplus}\right)\left(\cos \chi \sin \psi \sin \Omega_{\oplus} T_{\oplus}-\cos \psi \cos \Omega_{\oplus} T_{\oplus}\right) \\
& -2 c_{f}^{X Z} \sin \chi \sin \psi\left(\cos \chi \sin \psi \cos \Omega_{\oplus} T_{\oplus}+\cos \psi \sin \Omega_{\oplus} T_{\oplus}\right) \\
& +2 c_{f}^{Y Z} \sin \chi \sin \psi\left(\cos \chi \sin \psi \sin \Omega_{\oplus} T_{\oplus}-\cos \psi \cos \Omega_{\oplus} T_{\oplus}\right)+c_{f}^{Z Z} \sin ^{2} \chi \sin ^{2} \psi
\end{aligned}
$$

The reader is reminded that $\chi$ is the laboratory colatitude, $\psi$ is the angle north of east specifying the beam orientation, and $\Omega_{\oplus} T_{\oplus}$ is the local sidereal angle.

Note that the first line of the expression (4.18) represents the conventional result shifted by the factor $\left(1+c_{f}^{33}-c_{f}^{00}\right)$, which stems from the modified partonic subprocess $q \bar{q} \rightarrow \gamma \rightarrow l \bar{l}$ encapsulated in eq. (4.16). The remainder arises from the shifted argument in the delta function, leading to additional kinematical dependence and derivatives of the PDFs themselves. In the conventional case, the quantity $Q^{4} d \sigma / d Q^{2}$ exhibits a scaling law in that it is a function only of $1 / \tau=s / Q^{2}$. This scaling law persists at tree level in the DY process in the presence of Lorentz violation. In contrast, the $c$-type coefficients induce scaling violations in DIS.

\subsection{Nonminimal $a^{(5)}$-type coefficients}

Next, we revisit the effect of nonzero $a^{(5)}$-type coefficients on the unpolarized scattering DY process. The effects of the corresponding CPT-violating operators on the partonantiparton collision has some interesting features. The same PDFs $f_{f}(\xi), f_{\bar{f}}(\xi)$ emerge as in the analysis for $c$-type coefficients because the $a^{(5)}$-type coefficients also control spinindependent effects. Using the Feynman rules and noting eqs. (3.51) and (3.53), we again find $W_{f}^{\mu \nu}$ takes the form (4.11). The perturbative contribution is now given by eq. (4.12) with the replacements

$$
\begin{aligned}
& \left(\eta^{\mu \alpha}+c_{f}^{\alpha \mu}\right) \gamma_{\alpha} \rightarrow\left(\eta^{\mu \alpha}-a_{\mathrm{S} f}^{(5) \alpha \beta \mu}\right) \gamma_{\alpha}\left(\xi_{1} p_{1}+\xi_{2} p_{2}\right)_{\beta}, \\
& \left(\eta^{\mu \alpha}+c_{f}^{\mu \alpha}\right) q_{\alpha} \rightarrow q^{\mu} \mp a_{\mathrm{S} f}^{(5) \mu \alpha \beta} \widetilde{k}_{1_{\alpha}} \widetilde{k}_{1_{\beta}} \pm a_{\mathrm{S} f}^{(5) \mu \alpha \beta} \widetilde{k}_{2_{\alpha}} \widetilde{k}_{2_{\beta}} .
\end{aligned}
$$

The upper signs in the latter expression hold for $k_{1}$ associated to a particle and $k_{2}$ to an antiparticle, while the lower signs hold for $k_{1}$ associated to an antiparticle and $k_{2}$ to a particle. The hard scattering functions $H_{f}^{\mu \nu}\left(\widetilde{k}_{1}^{+}, \widetilde{k}_{2}^{-}\right)$and $H_{f}^{\mu \nu}\left(\widetilde{k}_{2}^{-}, \widetilde{k}_{1}^{+}\right)$now differ because $\widetilde{q}$ is asymmetric under the interchange $\widetilde{k}_{1} \leftrightarrow \widetilde{k}_{2}$ due to the opposite-sign contributions from the $a^{(5)}$-type coefficients for quarks and antiquarks. The two contributions are therefore distinct, and the hadronic tensor takes the factorized form

$$
W_{f}^{\mu \nu}=\int d \xi_{1} d \xi_{2}\left[H_{f}^{\mu \nu}\left(\xi_{1}, \xi_{2}\right) f_{f}\left(\xi_{1}\right) f_{\bar{f}}\left(\xi_{2}\right)+H_{f}^{\mu \nu}\left(\xi_{2}, \xi_{1}\right) f_{f}\left(\xi_{2}\right) f_{\bar{f}}\left(\xi_{1}\right)\right] .
$$


However, this has little relevance for the total cross section, as integrating over the entire available phase space gives identical contributions in each case. Explicitly, we find for the total cross section

$$
\begin{aligned}
\sigma=\frac{2 \alpha^{2}}{3 s} \frac{1}{Q^{4}} \sum_{f} e_{f}^{2} \int & d \Omega_{l} \frac{d x_{1}}{x_{1}} \frac{d x_{2}}{x_{2}}\left[\left(\widetilde{k}_{1} \cdot l_{1}\right)\left(\widetilde{k}_{2} \cdot l_{2}\right)+\left(\widetilde{k}_{1} \cdot l_{2}\right)\left(\widetilde{k}_{2} \cdot l_{1}\right)\right. \\
& +\left(\widetilde{k}_{1} \cdot \widetilde{k}_{2}\right)\left(a_{\mathrm{S} f}^{(5) l_{1} \widetilde{k}_{1} l_{2}}+a_{\mathrm{S} f}^{(5) l_{1} \widetilde{k}_{2} l_{2}}+\left(l_{1} \leftrightarrow l_{2}\right)\right) \\
& -\left(\left(\left(\widetilde{k}_{1} \cdot l_{1}\right)\left(a_{\mathrm{S} f}^{(5) \widetilde{k}_{2} \widetilde{k}_{1} l_{2}}+a_{\mathrm{S} f}^{(5) \widetilde{k}_{2} \widetilde{k}_{2} l_{2}}+\left(l_{1} \leftrightarrow l_{2}\right)\right)\right)+\left(\widetilde{k}_{1} \leftrightarrow \widetilde{k}_{2}\right)\right) \\
& \left.+\left(l_{1} \cdot l_{2}\right)\left(a_{\mathrm{S} f}^{(5) \widetilde{k}_{1} \widetilde{k}_{1} \widetilde{k}_{2}}+a_{\mathrm{S} f}^{(5) \widetilde{k}_{1} \widetilde{k}_{2} \widetilde{k}_{2}}+a_{\mathrm{S} f}^{(5) \widetilde{k}_{2} \widetilde{k}_{1} \widetilde{k}_{1}}+a_{\mathrm{S} f}^{\left.(5) \widetilde{k}_{2} \widetilde{k}_{2} \widetilde{k}_{1}\right)}\right)\right] \\
\times & \left(f_{f}\left(x_{1},+\right) f_{\bar{f}}\left(x_{2},-\right)+f_{f}\left(x_{2},+\right) f_{\bar{f}}\left(x_{1},-\right)\right) .
\end{aligned}
$$

Here, we employ the notation $f_{f}(x, \pm)$ and $f_{\bar{f}}(x, \pm)$ to denote the sign dependences on the $a^{(5)}$-type scalar quantities that may appear in the PDFs, as discussed in section 3.4.

The differential distribution $d \sigma / d Q^{2}$ in terms of $\widehat{s}=\left(k_{1}+k_{2}\right)^{2}$ is required. At first order in Lorentz violation, it takes the general form

$$
\widehat{s}_{ \pm}=2 \widetilde{k}_{1} \cdot \widetilde{k}_{2} \pm 2\left(a_{\mathrm{S} f}^{(5) \widetilde{k}_{1} \widetilde{k}_{1} \widetilde{k}_{1}}-a_{\mathrm{S} f}^{(5) \widetilde{k}_{2} \widetilde{k}_{2} \widetilde{k}_{2}}-a_{\mathrm{S} f}^{(5) \widetilde{k}_{1} \widetilde{k}_{2} \widetilde{k}_{2}}+a_{\mathrm{S} f}^{(5) \widetilde{k}_{2} \widetilde{k}_{1} \widetilde{k}_{1}}\right),
$$

where the upper sign is for the particle with $k_{1}$ and the lower sign for the antiparticle. Using the CM-frame kinematics for the DY process, we obtain

$$
\begin{aligned}
\widehat{s}_{ \pm}=s x_{1} x_{2} \pm s E_{p} & {\left[\frac{1}{2} a_{\mathrm{S} f}^{(5) 000}\left(x_{1}-x_{2}\right)\left(x_{1}+x_{2}\right)^{2}-a_{\mathrm{S} f}^{(5) 003}\left(x_{1}+x_{2}\right)\left(x_{1}^{2}+x_{2}^{2}\right)\right.} \\
& +\frac{1}{2} a_{\mathrm{S} f}^{(5) 033}\left(x_{1}-x_{2}\right)\left(x_{1}+x_{2}\right)^{2}-\frac{1}{2} a_{\mathrm{S} f}^{(5) 300}\left(x_{1}+x_{2}\right)\left(x_{1}-x_{2}\right)^{2} \\
& \left.+a_{\mathrm{S} f}^{(5) 330}\left(x_{1}-x_{2}\right)\left(x_{1}^{2}+x_{2}^{2}\right)-\frac{1}{2} a_{\mathrm{S} f}^{(5) 333}\left(x_{1}+x_{2}\right)\left(x_{1}-x_{2}\right)^{2}\right] .
\end{aligned}
$$

Like the hard-scattering trace, this expression has symmetric and antisymmetric pieces in $x_{1}, x_{2}$. This differs from the result for the $c$-type coefficients, where the hard-scattering trace is symmetric and so only the symmetric parts of $\widehat{s}$ contribute.

Carrying out the calculation as before, we find

$$
\begin{aligned}
\frac{d \sigma}{d Q^{2}}=\frac{4 \pi \alpha^{2}}{9 Q^{4}} \sum_{f} e_{f}^{2} \int_{0}^{1} d x & {\left[\frac{\tau}{x}\left(1+A_{\mathrm{S}}(x, \tau / x)\right) f_{\mathrm{S} f}(x, \tau / x)\right.} \\
& \left.-\frac{\tau}{s x^{2}}\left(A_{\mathrm{A}}^{\prime}(x, \tau / x) f_{\mathrm{A} f}(x, \tau / x)+A_{\mathrm{A}}(x, \tau / x) f_{\mathrm{A} f}^{\prime}\right)\right],
\end{aligned}
$$

where

$$
\begin{aligned}
f_{\mathrm{S} f}(x, \tau / x) & \equiv f_{f}(x) f_{\bar{f}}(\tau / x)+f_{f}(\tau / x) f_{\bar{f}}(x), \\
f_{\mathrm{A} f}(x, \tau / x) & \equiv f_{f}(x) f_{\bar{f}}(\tau / x)-f_{f}(\tau / x) f_{\bar{f}}(x), \\
f_{\mathrm{A} f}^{\prime}(x, \tau / x) & \equiv f_{f}(x) f_{\bar{f}}^{\prime}(\tau / x)-f_{f}^{\prime}(\tau / x) f_{\bar{f}}(x), \\
A_{\mathrm{S}} & =E_{p}(x+\tau / x)\left(a_{\mathrm{S} f}^{(5) 110}+a_{\mathrm{S} f}^{(5) 220}\right),
\end{aligned}
$$


and

$$
\begin{aligned}
A_{\mathrm{A}}(x, \tau / x)=s E_{p}[ & \frac{1}{2}(x-\tau / x)(x+\tau / x)^{2}\left(a_{\mathrm{S} f}^{(5) 000}+a_{\mathrm{S} f}^{(5) 033}\right) \\
+ & \left.a_{\mathrm{S} f}^{(5) 330}(x-\tau / x)\left(x^{2}+(\tau / x)^{2}\right)\right], \\
A_{\mathrm{A}}^{\prime}(x, \tau / x)=-\frac{s}{2 x^{2}} E_{p} & {\left[2\left(x^{4}-2 \tau x^{2}+3 \tau^{2}\right) a_{\mathrm{S} f}^{(5) 330}\right.} \\
& \left.-\left(x^{2}-3 \tau\right)\left(x^{2}+\tau\right)\left(a_{\mathrm{S} f}^{(5) 000}+a_{\mathrm{S} f}^{(5) 033}\right)\right] .
\end{aligned}
$$

The first line of the result (4.25) represents a modification to the conventional result that is symmetric in $x_{1}$ and $x_{2}$. The analogous result for $c$-type coefficients involves a shift given by $c_{f}^{33}-c_{f}^{00}=c_{f}^{11}+c_{f}^{22}$ once trace considerations are taken into account, which has similarities with the combination of coefficients found in eq. (4.27). The remaining terms result from the shifted delta function and the combinations antisymmetric in $x_{1}$ and $x_{2}$. Note also that the PDFs derived here are the same as those found in DIS. One new feature is that scaling violations are present by virtue of the mass dimensionality of the $a^{(5)}$-type coefficients. Also, since the DY process is more symmetric than DIS, a smaller set of coefficients for Lorentz violation appears in the cross section (4.25).

\subsection{Estimated attainable sensitivities and comparison with DIS}

In this section, we present estimated attainable sensitivities extracted from $d \sigma / d Q^{2}$ measurements of the DY process at the LHC and discuss the relative advantages of searches using DIS and the DY process. For definiteness, we consider CMS results for the DY process in the dielectron channel as presented in ref. [13]. These data involve a CM energy of $\sqrt{s}=13 \mathrm{TeV}$ with a dielectron invariant mass of up to $Q^{2}=60 \mathrm{GeV}$, which lies safely below the $Z$ pole. They involve nine bins of width $5 \mathrm{GeV}$ starting at $15 \mathrm{GeV}$. The colatitude of CMS is $\chi \approx 46^{\circ}$, and the orientation of the beamline is $\psi \approx-14^{\circ}$. With these values, applying the appropriate rotation matrices yields the relevant combinations of coefficients in the Sun-centered frame that affect the cross sections. We use the $d \sigma / d Q^{2}$ form of the cross sections for $c$ - and $a^{(5)}$-type coefficients as given by eqs. (4.18) and (4.24), respectively, and evaluate them at the median value of each $Q^{2}$ bin.

Adopting a simulation strategy analogous to that for DIS in the case of purely uncorrelated systematic uncertainties, we list in table 3 the extracted estimated attainable sensitivities for both $c$ - and $a^{(5)}$-type coefficients. Note that the set of coefficients affecting the DY process is smaller than that affecting DIS, which leads to fewer coefficient combinations controlling sidereal-time dependence and hence fewer independent sensitivities. For the $c$-type coefficients for the $u$ and $d$ quarks, the strongest estimated sensitivities are found to come from the lowest $Q^{2}$ bin and lie in the range $10^{-5}-10^{-3}$. For the $a^{(5)}$-type coefficients, the best estimated sensitivities again arise from the lowest $Q^{2}$ bin and lie in the range $10^{-9}-10^{-7} \mathrm{GeV}^{-1}$. The emergence of greater sensitivities at lower $Q^{2}$ and larger $\mathrm{CM}$ energy can be expected from the structure of the cross sections.

It is interesting to compare the attainable sensitivities to Lorentz violation in DIS and the DY process. Table 4 displays the estimated attainable sensitivities from DIS at 


\begin{tabular}{|c|c|}
\hline & LHC \\
\hline$\left|c_{u}^{X Z}\right|$ & $7.3[19]$ \\
$\left|c_{u}^{Y Z}\right|$ & $7.1[19]$ \\
$\left|c_{u}^{X Y}\right|$ & $2.7[7.0]$ \\
$\left|c_{u}^{X X}-c_{u}^{Y Y}\right|$ & $15[39]$ \\
\hline$\left|c_{d}^{X Z}\right|$ & $72[180]$ \\
$\left|c_{d}^{Y Z}\right|$ & $70[180]$ \\
$\left|c_{d}^{X Y}\right|$ & $26[69]$ \\
$\left|c_{d}^{X X}-c_{d}^{Y Y}\right|$ & $150[400]$ \\
\hline$\left|a_{\mathrm{Su}}^{(5) T X X}-a_{\mathrm{Su}}^{(5) T Y Y}\right|$ & $0.015[0.039]$ \\
$\left|a_{\mathrm{S} u}^{(5) T X Y}\right|$ & $0.0027[0.0070]$ \\
$\left|a_{\mathrm{S} u}^{(5) T X Z}\right|$ & $0.0072[0.019]$ \\
$\left|a_{\mathrm{S} u}^{(5) T Y Z}\right|$ & $0.0070[0.018]$ \\
\hline$\left|a_{\mathrm{S} d}^{(5) T X X}-a_{\mathrm{S} d}^{(5) T Y Y}\right|$ & $0.19[0.49]$ \\
$\left|a_{\mathrm{S} d}^{(5) T X Y}\right|$ & $0.034[0.088]$ \\
$\left|a_{\mathrm{S} d}^{(5) T X Z}\right|$ & $0.090[0.23]$ \\
$\left|a_{\mathrm{S} d}^{(5) T Y Z}\right|$ & $0.089[0.23]$ \\
\hline
\end{tabular}

Table 3. Expected best sensitivities on individual coefficients $c_{f}^{J K}$ and $a_{\mathrm{S} f}^{(5) T K}$ from studies of the DY process at the LHC. Values are in units of $10^{-5}$ and $10^{-6} \mathrm{GeV}^{-1}$, respectively. Results with brackets are associated with uncorrelated systematic uncertainties between binned data, while results without brackets correspond to the assumption of $100 \%$ correlation between systematic uncertainties.

\begin{tabular}{|c|c|c|}
\hline & EIC & LHC \\
\hline$\left|c_{u}^{X X}-c_{u}^{Y Y}\right|$ & 0.37 & 15 \\
$\left|c_{u}^{X Y}\right|$ & 0.13 & 2.7 \\
$\left|c_{u}^{X Z}\right|$ & 0.11 & 7.3 \\
$\left|c_{u}^{Y Z}\right|$ & 0.12 & 7.1 \\
\hline$\left|a_{\mathrm{S} u}^{(5) T X X}-a_{\mathrm{Su}}^{(5) T Y Y}\right|$ & 2.3 & 0.015 \\
$\left|a_{\mathrm{Su}}^{(5) T X Y}\right|$ & 0.34 & 0.0027 \\
$\left|a_{\mathrm{S} u}^{(5) T X Z}\right|$ & 0.13 & 0.0072 \\
$\left|a_{\mathrm{S} u}^{(5) T Y Z}\right|$ & 0.12 & 0.0070 \\
\hline
\end{tabular}

Table 4. Comparison of estimated attainable sensitivities to equivalent $u$-quark coefficients at the EIC and the LHC. Values are in units of $10^{-5}$ and $10^{-6} \mathrm{GeV}^{-1}$ for the minimal and nonminimal coefficients, respectively. 
the EIC and from the DY process at the LHC for the $u$-quark coefficient combinations that contribute to sidereal-time variations in both experiments. The prospective LHC sensitivities are weaker by an order of magnitude for minimal $c$-type coefficients, due to the dominance of the small statistical uncertainties at the EIC. In contrast, the prospective LHC sensitivities are better by an order of magnitude for the $a^{(5)}$-type coefficients, due primarily to the larger CM energy. The latter result supports the notion that higherenergy colliders have a comparative advantage in constraining coefficients with negative mass dimension since the dimensionless quantity measured in experiments is essentially the product of the coefficient and the collider energy. Given the current lack of direct constraints in the strongly interacting sector of the SME [11], all these results offer strong encouragement for searches for Lorentz and CPT violation in a variety of processes and using distinct collider experiments.

\section{Summary}

In this work, we have performed a theoretical and phenomenological exploration of the effects of Lorentz and CPT violation in high-energy hadronic processes. The equivalent parton-model picture is derived in the presence of effects on freely propagating quarks emanating from the modified factorization procedure of the hadronic tensor in inclusive DIS. This leads to new definitions of the leading-twist PDFs and for the first time parametrizes and explains the potential nonperturbative dependence on Lorentz violation. The validity of this general treatment is confirmed using the alternative approach of the operator product expansion and via the electromagnetic Ward identities. Factorization is also demonstrated in the DY process. The PDFs derived for the DY process are identical to those found in DIS, supporting the conjecture that universality of the PDFs can be retained despite the presence of Lorentz violation.

The phenomenological implications of this framework are explored by considering the special cases of unpolarized electron-proton DIS and the DY process mediated by photon exchange for the minimal $c$-type and nonminimal $a^{(5)}$-type coefficients. Our results show that searches for Lorentz violation at lepton-hadron and hadron-hadron colliders are complementary. The methodology presented in the present work opens the path for future studies of a multitude of related processes, including charged-current, polarized leptonhadron, and hadron-hadron interactions, as well as investigations of higher-order effects such as QCD corrections.

\section{Acknowledgments}

This work was supported in part by the U.S. Department of Energy under grant DESC0010120, by the Indiana Space Grant Consortium, and by the Indiana University Center for Spacetime Symmetries.

Open Access. This article is distributed under the terms of the Creative Commons Attribution License (CC-BY 4.0), which permits any use, distribution and reproduction in any medium, provided the original author(s) and source are credited. 


\section{References}

[1] E.D. Bloom et al., High-Energy Inelastic e p Scattering at 6-Degrees and 10-Degrees, Phys. Rev. Lett. 23 (1969) 930 [INSPIRE].

[2] M. Breidenbach et al., Observed Behavior of Highly Inelastic electron-Proton Scattering, Phys. Rev. Lett. 23 (1969) 935 [inSPIRE].

[3] J.D. Bjorken, Asymptotic Sum Rules at Infinite Momentum, Phys. Rev. 179 (1969) 1547 [INSPIRE].

[4] R.P. Feynman, Very high-energy collisions of hadrons, Phys. Rev. Lett. 23 (1969) 1415 [INSPIRE].

[5] S.D. Drell and T.-M. Yan, Massive Lepton Pair Production in Hadron-Hadron Collisions at High-Energies, Phys. Rev. Lett. 25 (1970) 316 [Erratum ibid. 25 (1970) 902] [INSPIRE].

[6] J.H. Christenson, G.S. Hicks, L.M. Lederman, P.J. Limon, B.G. Pope and E. Zavattini, Observation of massive muon pairs in hadron collisions, Phys. Rev. Lett. 25 (1970) 1523 [INSPIRE].

[7] SISSA, Proceedings, 26th International Workshop on Deep Inelastic Scattering and Related Subjects (DIS 2018), vol. DIS2018, SISSA, Port Island, Kobe, Japan, 16-20 April 2018 [INSPIRE].

[8] V.A. Kostelecký and S. Samuel, Spontaneous Breaking of Lorentz Symmetry in String Theory, Phys. Rev. D 39 (1989) 683 [InSPIRE].

[9] V.A. Kostelecký and R. Potting, CPT and strings, Nucl. Phys. B 359 (1991) 545 [INSPIRE].

[10] V.A. Kostelecký and R. Potting, CPT, strings and meson factories, Phys. Rev. D 51 (1995) 3923 [hep-ph/9501341] [INSPIRE].

[11] V.A. Kostelecký and N. Russell, Data Tables for Lorentz and CPT Violation, Rev. Mod. Phys. 83 (2011) 11 [arXiv:0801.0287] [INSPIRE].

[12] H1 and ZEUS collaborations, Combination of measurements of inclusive deep inelastic $e^{ \pm} p$ scattering cross sections and QCD analysis of HERA data, Eur. Phys. J. C $\mathbf{7 5}$ (2015) 580 [arXiv: 1506.06042] [INSPIRE].

[13] CMS collaboration, Measurement of the differential Drell-Yan cross section in proton-proton collisions at $\sqrt{\mathrm{s}}=13 \mathrm{TeV}$, JHEP 12 (2019) 059 [arXiv:1812.10529] [INSPIRE].

[14] S. Weinberg, Effective Field Theory, Past and Future, PoS(CD09)001 (2009) [arXiv:0908.1964] [INSPIRE].

[15] D. Colladay and V.A. Kostelecký, CPT violation and the standard model, Phys. Rev. D 55 (1997) 6760 [hep-ph/9703464] [INSPIRE].

[16] D. Colladay and V.A. Kostelecký, Lorentz violating extension of the standard model, Phys. Rev. D 58 (1998) 116002 [hep-ph/9809521] [INSPIRE].

[17] V.A. Kostelecký, Gravity, Lorentz violation and the standard model, Phys. Rev. D 69 (2004) 105009 [hep-th/0312310] [INSPIRE].

[18] O.W. Greenberg, CPT violation implies violation of Lorentz invariance, Phys. Rev. Lett. 89 (2002) 231602 [hep-ph/0201258] [INSPIRE].

[19] R. Bluhm, Overview of the SME: Implications and phenomenology of Lorentz violation, Lect. Notes Phys. 702 (2006) 191 [hep-ph/0506054] [INSPIRE]. 
[20] J.D. Tasson, What Do We Know About Lorentz Invariance?, Rept. Prog. Phys. 77 (2014) 062901 [arXiv: 1403.7785] [INSPIRE].

[21] A. Hees, Q.G. Bailey, A. Bourgoin, H. P.-L. Bars, C. Guerlin and C. Le Poncin-Lafitte, Tests of Lorentz symmetry in the gravitational sector, Universe 2 (2016) 30 [arXiv:1610.04682] [INSPIRE].

[22] D0 collaboration, Search for violation of Lorentz invariance in top quark pair production and decay, Phys. Rev. Lett. 108 (2012) 261603 [arXiv:1203.6106] [INSPIRE].

[23] M.S. Berger, V.A. Kostelecký and Z. Liu, Lorentz and CPT Violation in Top-Quark Production and Decay, Phys. Rev. D 93 (2016) 036005 [arXiv: 1509.08929] [InSPIRE].

[24] A. Carle, N. Chanon and S. Perries, Prospects for Lorentz Invariance Violation searches with top pair production at the LHC and future hadron colliders, Eur. Phys. J. C 80 (2020) 128 [arXiv: 1908.11256] [INSPIRE].

[25] R. Kamand, B. Altschul and M.R. Schindler, Hadronic Lorentz Violation in Chiral Perturbation Theory, Phys. Rev. D 95 (2017) 056005 [arXiv: 1608.06503] [INSPIRE].

[26] R. Kamand, B. Altschul and M.R. Schindler, Hadronic Lorentz Violation in Chiral Perturbation Theory Including the Coupling to External Fields, Phys. Rev. D 97 (2018) 095027 [arXiv: 1712.00838] [INSPIRE].

[27] B. Altschul and M.R. Schindler, Lorentz- and CPT-violating standard model extension in chiral perturbation theory, Phys. Rev. D 100 (2019) 075031 [arXiv: 1907. 02490] [INSPIRE].

[28] J.P. Noordmans, J. de Vries and R.G.E. Timmermans, Tests of Lorentz and CPT symmetry with hadrons and nuclei, Phys. Rev. C 94 (2016) 025502 [arXiv:1602.00496] [INSPIRE].

[29] J.P. Noordmans, Gluonic Lorentz violation and chiral perturbation theory, Phys. Rev. D 95 (2017) 075030 [arXiv: 1701.04334] [INSPIRE].

[30] V.A. Kostelecký, E. Lunghi and A.R. Vieira, Lorentz violation and deep inelastic scattering, Phys. Lett. B 769 (2017) 272 [arXiv:1610.08755] [INSPIRE].

[31] E. Lunghi and N. Sherrill, Lorentz violation and the electron-ion collider, Phys. Rev. D 98 (2018) 115018 [arXiv:1805.11684] [INSPIRE].

[32] V.A. Kostelecký and Z. Li, Gauge field theories with Lorentz-violating operators of arbitrary dimension, Phys. Rev. D 99 (2019) 056016 [arXiv:1812.11672] [INSPIRE].

[33] I.S. Karpikov, D. Tlisov and D. Kirpichnikov, Space anisotropy search at colliders, Phys. Atom. Nucl. 81 (2018) 257 [arXiv:1612.02217] [INSPIRE].

[34] A. Michel and M. Sher, Breaking of Lorentz Invariance in Electron-Proton Parity Violation, Phys. Rev. D 100 (2019) 095011 [arXiv:1909.10627] [INSPIRE].

[35] V.A. Kostelecký, Sensitivity of CPT tests with neutral mesons, Phys. Rev. Lett. 80 (1998) 1818 [hep-ph/9809572] [INSPIRE].

[36] B.R. Edwards and V.A. Kostelecký, Searching for CPT Violation with Neutral-Meson Oscillations, Phys. Lett. B 795 (2019) 620 [arXiv:1907.05206] [INSPIRE].

[37] V.A. Kostelecký, Signals for CPT and Lorentz violation in neutral meson oscillations, Phys. Rev. D 61 (2000) 016002 [hep-ph/9909554] [INSPIRE].

[38] V.A. Kostelecký and A. Roberts, Analog models for $T$ and CPT violation in neutral meson oscillations, Phys. Rev. D 63 (2001) 096002 [hep-ph/0012381] [INSPIRE]. 
[39] N. Isgur, V.A. Kostelecký and A.P. Szczepaniak, Background enhancement of CPT reach at an asymmetric phi factory, Phys. Lett. B 515 (2001) 333 [hep-ph/0106353] [INSPIRE].

[40] H. Nguyen, CPT Results from KTeV, in CPT and Lorentz symmetry. Proceedings: 2nd Meeting, Bloomington, U.S.A., 15-18 August 2001, pp. 122-131 (2002) [DOI] [hep-ex/0112046] [INSPIRE].

[41] KLOE-2 collaboration, Test of CPT and Lorentz symmetry in entangled neutral kaons with the KLOE experiment, Phys. Lett. B 730 (2014) 89 [arXiv:1312.6818] [INSPIRE].

[42] V.A. Kostelecký, Formalism for CPT, T and Lorentz violation in neutral meson oscillations, Phys. Rev. D 64 (2001) 076001 [hep-ph/0104120] [InSPIRE].

[43] BABAR collaboration, Search for CPT and Lorentz violation in $B^{0}-\bar{B}^{0}$ oscillations with dilepton events, Phys. Rev. Lett. 100 (2008) 131802 [arXiv:0711.2713] [INSPIRE].

[44] A. Kostelecký and R. Van Kooten, CPT violation and B-meson oscillations, Phys. Rev. D 82 (2010) 101702 [arXiv: 1007.5312] [INSPIRE].

[45] D0 collaboration, Search for Violation of CPT and Lorentz Invariance in $B_{s}^{0}$ Meson Oscillations, Phys. Rev. Lett. 115 (2015) 161601 [arXiv:1506.04123] [INSPIRE].

[46] LHCb collaboration, Search for violations of Lorentz invariance and CPT symmetry in $B_{(s)}^{0}$ mixing, Phys. Rev. Lett. 116 (2016) 241601 [arXiv: 1603.04804] [INSPIRE].

[47] Á. Roberts, Testing CPT symmetry with correlated neutral mesons, Phys. Rev. D 96 (2017) 116015 [arXiv: 1706.03378] [INSPIRE].

[48] K.R. Schubert, Tests of CPT symmetry in B0-B0bar mixing and in B0 to c cbar K0 decays, in Proceedings, 7th Meeting on CPT and Lorentz Symmetry (CPT 16), Bloomington, Indiana, U.S.A., 20-24 June 2016, pp. 121-124 (2017) [DOI] [arXiv:1607.05882] [INSPIRE].

[49] O. Bertolami, D. Colladay, V.A. Kostelecký and R. Potting, CPT violation and baryogenesis, Phys. Lett. B 395 (1997) 178 [hep-ph/9612437] [INSPIRE].

[50] E. Di Grezia, S. Esposito and G. Salesi, Baryon asymmetry in the universe resulting from Lorentz violation, Europhys. Lett. 74 (2006) 747 [hep-ph/0508298] [INSPIRE].

[51] C.M. Ho, Leptogenesis and CPT Violation, Phys. Lett. B 702 (2011) 398 [arXiv:1012.1053] [INSPIRE].

[52] N.E. Mavromatos and S. Sarkar, Spontaneous CPT Violation and Quantum Anomalies in a Model for Matter-Antimatter Asymmetry in the Cosmos, Universe 5 (2018) 5 [arXiv: 1812.00504] [INSPIRE].

[53] A.D. Sakharov, Violation of CP Invariance, $C$ asymmetry and baryon asymmetry of the universe, Pisma Zh. Eksp. Teor. Fiz. 5 (1967) 32 [inSPIRE].

[54] A. Kostelecký and M. Mewes, Fermions with Lorentz-violating operators of arbitrary dimension, Phys. Rev. D 88 (2013) 096006 [arXiv: 1308.4973] [INSPIRE].

[55] A. Kostelecký and M. Mewes, Neutrinos with Lorentz-violating operators of arbitrary dimension, Phys. Rev. D 85 (2012) 096005 [arXiv:1112.6395] [INSPIRE].

[56] A.H. Gomes, A. Kostelecký and A.J. Vargas, Laboratory tests of Lorentz and CPT symmetry with muons, Phys. Rev. D 90 (2014) 076009 [arXiv:1407.7748] [INSPIRE].

[57] V.A. Kostelecký and A.J. Vargas, Lorentz and CPT tests with hydrogen, antihydrogen and related systems, Phys. Rev. D 92 (2015) 056002 [arXiv: 1506.01706] [INSPIRE]. 
[58] M. Schreck, Classical Lagrangians and Finsler structures for the nonminimal fermion sector of the Standard-Model Extension, Phys. Rev. D 93 (2016) 105017 [arXiv:1512.04299] [INSPIRE].

[59] V.A. Kostelecký and A.J. Vargas, Lorentz and CPT Tests with Clock-Comparison Experiments, Phys. Rev. D 98 (2018) 036003 [arXiv: 1805.04499] [InSPIRE].

[60] ICECuBE collaboration, Neutrino Interferometry for High-Precision Tests of Lorentz Symmetry with IceCube, Nature Phys. 14 (2018) 961 [arXiv:1709.03434] [INSPIRE].

[61] J. Collins, Foundations of perturbative QCD, Camb. Monogr. Part. Phys. Nucl. Phys. Cosmol. 32 (2011) 1 [INSPIRE].

[62] J.C. Collins, D.E. Soper and G.F. Sterman, Factorization of Hard Processes in QCD, Adv. Ser. Direct. High Energy Phys. 5 (1989) 1 [hep-ph/0409313] [INSPIRE].

[63] V.A. Kostelecký and R. Lehnert, Stability, causality and Lorentz and CPT violation, Phys. Rev. D 63 (2001) 065008 [hep-th/0012060] [INSPIRE].

[64] D. Colladay and P. McDonald, Redefining spinors in Lorentz violating QED, J. Math. Phys. 43 (2002) 3554 [hep-ph/0202066] [INSPIRE].

[65] B. Altschul, Eliminating the CPT-odd $f$ coefficient from the Lorentz-violating standard model extension, J. Phys. A 39 (2006) 13757 [hep-th/0602235] [INSPIRE].

[66] R. Lehnert, Non-local on-shell field redefinition for the SME, Phys. Rev. D 74 (2006) 125001 [hep-th/0609162] [INSPIRE].

[67] A.V. Kostelecký and J.D. Tasson, Matter-gravity couplings and Lorentz violation, Phys. Rev. D 83 (2011) 016013 [arXiv: 1006.4106] [INSPIRE].

[68] Y. Bonder, Lorentz violation in the gravity sector: The t puzzle, Phys. Rev. D 91 (2015) 125002 [arXiv: 1504.03636] [INSPIRE].

[69] Y. Ding and V.A. Kostelecký, Lorentz-violating spinor electrodynamics and Penning traps, Phys. Rev. D 94 (2016) 056008 [arXiv: 1608.07868] [INSPIRE].

[70] J. Foster, V.A. Kostelecký and R. Xu, Constraints on Nonmetricity from Bounds on Lorentz Violation, Phys. Rev. D 95 (2017) 084033 [arXiv:1612.08744] [INSPIRE].

[71] A.V. Kostelecký and N. Russell, Classical kinematics for Lorentz violation, Phys. Lett. B 693 (2010) 443 [arXiv: 1008.5062] [INSPIRE].

[72] A. Kostelecký, Riemann-Finsler geometry and Lorentz-violating kinematics, Phys. Lett. B 701 (2011) 137 [arXiv:1104.5488] [INSPIRE].

[73] B.R. Edwards and V.A. Kostelecký, Riemann-Finsler geometry and Lorentz-violating scalar fields, Phys. Lett. B 786 (2018) 319 [arXiv:1809.05535] [INSPIRE].

[74] M. Schreck, Classical Lagrangians for the nonminimal Standard-Model Extension at higher orders in Lorentz violation, Phys. Lett. B 793 (2019) 70 [arXiv:1903.05064] [INSPIRE].

[75] J.E.G. Silva, R.V. Maluf and C.A.S. Almeida, Bipartite-Finsler symmetries, Phys. Lett. B 798 (2019) 135009 [arXiv:1907.11214] [INSPIRE].

[76] A.V. Manohar, An Introduction to spin dependent deep inelastic scattering, in Lake Louise Winter Institute: Symmetry and Spin in the Standard Model, Lake Louise, Alberta, Canada, 23-29 February 1992, pp. 1-46 (1992) [hep-ph/9204208] [INSPIRE].

[77] R.L. Jaffe, H. Meyer and G. Piller, Spin, twist and hadron structure in deep inelastic processes, in Lectures on QCD Springer, Lect. Notes Phys. 496 (1997) 178. 
[78] D. Colladay and V.A. Kostelecký, Cross-sections and Lorentz violation, Phys. Lett. B 511 (2001) 209 [hep-ph/0104300] [INSPIRE].

[79] A. Fittante and N. Russell, Fermion observables for Lorentz violation, J. Phys. G 39 (2012) 125004 [arXiv: 1210.2003] [INSPIRE].

[80] R. Bluhm, V.A. Kostelecký and N. Russell, CPT and Lorentz tests in Penning traps, Phys. Rev. D 57 (1998) 3932 [hep-ph/9809543] [INSPIRE].

[81] R. Lehnert, Dirac theory within the standard model extension, J. Math. Phys. 45 (2004) 3399 [hep-ph/0401084] [INSPIRE].

[82] M. Cambiaso, R. Lehnert and R. Potting, Asymptotic states and renormalization in Lorentz-violating quantum field theory, Phys. Rev. D 90 (2014) 065003 [arXiv:1401.7317] [INSPIRE].

[83] A. Accardi et al., Electron Ion Collider: The Next QCD Frontier, Eur. Phys. J. A 52 (2016) 268 [arXiv: 1212.1701] [INSPIRE].

[84] E.C. Aschenauer et al., eRHIC Design Study: An Electron-Ion Collider at BNL, arXiv: 1409.1633 [INSPIRE].

[85] S. Abeyratne et al., MEIC Design Summary, arXiv:1504.07961 [INSPIRE].

[86] S. Carrazza, C. Degrande, S. Iranipour, J. Rojo and M. Ubiali, Can New Physics hide inside the proton?, Phys. Rev. Lett. 123 (2019) 132001 [arXiv: 1905.05215] [INSPIRE].

[87] V.A. Kostelecký and M. Mewes, Signals for Lorentz violation in electrodynamics, Phys. Rev. D 66 (2002) 056005 [hep-ph/0205211] [INSPIRE].

[88] R. Bluhm, V.A. Kostelecký, C.D. Lane and N. Russell, Clock comparison tests of Lorentz and CPT symmetry in space, Phys. Rev. Lett. 88 (2002) 090801 [hep-ph/0111141] [INSPIRE].

[89] R. Bluhm, V.A. Kostelecký, C.D. Lane and N. Russell, Probing Lorentz and CPT violation with space based experiments, Phys. Rev. D 68 (2003) 125008 [hep-ph/0306190] [INSPIRE].

[90] V.A. Kostelecký, A.C. Melissinos and M. Mewes, Searching for photon-sector Lorentz violation using gravitational-wave detectors, Phys. Lett. B 761 (2016) 1 [arXiv:1608.02592] [INSPIRE].

[91] E. Godat, ManeParse: Mathematica Toolbox for PDF Uncertainties and Application to New Physics Searches, in Proceedings, Meeting of the APS Division of Particles and Fields (DPF 2015), Ann Arbor, Michigan, U.S.A., 4-8 August 2015 (2015) [arXiv:1510.06009] [INSPIRE].

[92] D.B. Clark, E. Godat and F.I. Olness, ManeParse: A Mathematica reader for Parton Distribution Functions, Comput. Phys. Commun. 216 (2017) 126 [arXiv:1605.08012] [INSPIRE].

[93] H.-L. Lai et al., New parton distributions for collider physics, Phys. Rev. D 82 (2010) 074024 [arXiv: 1007.2241] [INSPIRE].

[94] M. Bahr et al., HERWIG++ Physics and Manual, Eur. Phys. J. C 58 (2008) 639 [arXiv:0803.0883] [INSPIRE].

[95] J. Bellm et al., HERWIG 7.0/HERWIG++ 3.0 release note, Eur. Phys. J. C 76 (2016) 196 [arXiv: 1512.01178] [INSPIRE].

[96] J.-w. Qiu and G.F. Sterman, Power corrections in hadronic scattering. 1. Leading 1/Q $Q^{2}$ corrections to the Drell-Yan cross-section, Nucl. Phys. B 353 (1991) 105 [INSPIRE]. 